NBER WORKING PAPER SERIES

\title{
THE VALUE OF TIME: EVIDENCE FROM AUCTIONED CAB RIDES
}

\author{
Nicholas Buchholz \\ Laura Doval \\ Jakub Kastl \\ Filip Mat jka \\ Tobias Salz \\ Working Paper 27087 \\ http://www.nber.org/papers/w27087 \\ NATIONAL BUREAU OF ECONOMIC RESEARCH \\ 1050 Massachusetts Avenue \\ Cambridge, MA 02138 \\ May 2020
}

We thank Liftago for providing the data. We thank Juan Castillo Camillo, Gabriel Kreindler, Rob Porter, Stephen Redding, and seminar participants at FTC, Maryland, MIT, Ohio State, Penn, Princeton, Stanford, UCL, WashU and IO2 for useful comments. We are grateful for the financial support from the Transportation Economics in the 21st Century Initiative of the NBER and U.S. Department of Transportation. Kastl is grateful for the financial support of the NSF (SES-1352305). All remaining errors are ours. The views expressed herein are those of the authors and do not necessarily reflect the views of the National Bureau of Economic Research.

NBER working papers are circulated for discussion and comment purposes. They have not been peerreviewed or been subject to the review by the NBER Board of Directors that accompanies official NBER publications.

(C) 2020 by Nicholas Buchholz, Laura Doval, Jakub Kastl, Filip Mat jka, and Tobias Salz. All rights reserved. Short sections of text, not to exceed two paragraphs, may be quoted without explicit permission provided that full credit, including $(\odot$ notice, is given to the source. 
The Value of Time: Evidence From Auctioned Cab Rides

Nicholas Buchholz, Laura Doval, Jakub Kastl, Filip Mat jka, and Tobias Salz

NBER Working Paper No. 27087

May 2020, Revised August 2020

JEL No. L0

\begin{abstract}
We recover valuations of time using detailed data from a large ride-hail platform, where drivers bid on trips and consumers choose between a set of rides with different prices and waiting times. We estimate demand as a function of prices and waiting times and find that price elasticities are substantially higher than waiting-time elasticities. We show how these estimates can be mapped into values of time that vary by place, person, and time of day. We find that the value of time during non-work hours is $16 \%$ lower than during work hours. Most of the heterogeneity in the value of time, however, is explained by individual differences. We apply our estimates to study optimal time incentives in highway procurement. Standard industry practices, which set incentives based on a uniform value of time, lead to mis-priced time costs by up to ninety percent.
\end{abstract}

Nicholas Buchholz

Princeton University

nbuchholz@princeton.edu

Laura Doval

California Institute of Technology

Pasadena, CA 91125

laura@laura-doval.com

Jakub Kastl

Department of Economics

Princeton University

Princeton, NJ 08544-1021

and NBER

jkastl@princeton.edu
Filip Mat jka

CERGE-EI

Filip.Matejka@cerge-ei.cz

Tobias Salz

MIT Deparatment of Economics

77 Massachusetts Avenue, E52-404

Cambridge, MA 02139

and NBER

tsalz@mit.edu 


\section{Introduction}

Allocating time is an important aspect of many economic decisions. Since Becker (1965), economists have debated the extent to which the labor-leisure trade-off can be used to measure people's value of time. Credible measures of the value of time are useful for a variety of important policy decisions. For instance, they are a critical input to infrastructure planning, particularly in the transportation sector. A unique empirical challenge in measuring the value of time is that in many economic settings - unlike in labor markets - time is not always directly priced. In this paper we use data from auctioned cab rides to overcome this empirical challenge, allowing us to estimate how the value of time varies across individuals, across places, and across time-of-day within a large urban area.

Transportation markets are inherently ones in which consumers express their value of time: consider, for instance, the various decisions involved in the daily commute from home to work (Domencich and McFadden, 1975). In this context commuters might trade off cheaper-yet-slower off-peak trains against more expensive express trains; choose to leave earlier than their work requires to beat the rush hour; or choose between different service tiers in a ride-hailing platform, which involve different waiting times and prices. The choices made when faced with such trade-offs are therefore informative about the value of time, or the opportunity cost of time spent traveling.

We use detailed consumer choice data from Liftago, a large European ride-hailing application to measure the value of time. This platform uses a unique mechanism to allocate each ride through a rapid auction process in which nearby drivers bid on ride requests. Requesting consumers then choose between bids based on various characteristics. Most importantly, bids often involve trade-offs between price and waiting time, or the time it would take the taxi to pick up the customer. This is in contrast to platforms like Uber and Lyft, which employ "surge" pricing to equilibrate demand and supply. Under such mechanisms, consumers do not get to directly express preferences over prices and waiting times. We observe both consumers' individual choice sets as well as their ultimate selection for 1.9 million ride requests and 5.2 million bids.

Our first contribution is to provide a direct and clean measurement of consumers' willingness-to-pay to reduce waiting times. We use the variation in choice sets and choices to estimate a demand system that depends on both prices and waiting times. Demand measures of this form are of first-order importance for the provision of public transportation infrastructure. They are also critically important for the ride hail industry, where price and waiting time are the two key variables on which firms compete. As we elaborate below, our setting allows us to overcome some of the empirical challenges in 
simultaneously measuring preferences over both prices and waiting-time.

Our second contribution, building on the work of Small (1982), is to provide a conceptual framework for interpreting the disutility of waiting, and to demonstrate how the willingness-to-pay for waiting-time reductions can be used to recover the value of time. When consumers choose a shorter waiting time over a lower price, they reveal that the value of their time at the destination is greater than the value at the origin location. Intuitively, the willingness to pay for lower waiting times is simply the difference between the value of time at the destination and the value of time at the origin. In keeping with this interpretation we refer to the willingness to pay for waiting time reductions as the net value of time (abbreviated as NVOT). We formalize this mapping, demonstrate that the average value of time by place, individuals and time-of-day is identified, and show how it can be recovered with a simple moment-based estimator. Rich geographic detail allows us to further decompose the value of time and interpret the prevailing patterns.

Our demand results show that consumers respond substantially to changes in both price and waiting time. Price elasticities are four to ten times as large as waiting-time elasticities. We find that on average the net value of time, expressed as an hourly quantity, is $\$ 13.47$. However, there is large variation in these measures within the day and across space. From the overnight hours to the mid-day, the willingness to pay for lower waiting times approximately doubles. Geographic differences are estimated to have an order of magnitude difference from one extreme to another. Because our data includes panel identifiers for both passengers and drivers, we are able to credibly identify the individual-specific heterogeneity in both the elasticities and the implied NVOT. We rank individuals by their relative sensitivity to prices and wait times and find that the top quartile have NVOT measures about 3.5 times higher than the bottom quartile.

We use our estimates to investigate how much of the overall variation in the value of time is driven by differences across people, locations, and time of day. Our approach for this investigation is similar to a branch of the labor literature that decomposes differences in wage into firm and worker specific variation (Abowd et al. (1999)). Based on this decomposition we can then define high-value of time places and high-value of time people. This exercise provides a number of important insights. First, we find that almost eighty percent of the variation in the value of time is driven by differences across people. Once individual-specific variation is taken into account, the differences across places are relatively small. We also find that people who express a higher vot are not necessarily doing so for the same places. In fact, the relationship between high value of time people and high value of time places is slightly negative.

We also conduct a small survey of passengers to corroborate our measurements, which reveals that the estimated value of time during the beginning of a typical work 
day is very close to the mean wage in the survey sample. This finding provides a link between our results and the widely used wage measure, enabling us to better understand and extrapolate from wage-based value of time estimates.

To demonstrate the applicability of our results in a policy context, we use our value of time estimates as opportunity cost of traffic delays in order to inform the use of time incentives in a road infrastructure procurement setting. This exercise builds on existing literature studying the impact of time incentives in highway procurement (Lewis and Bajari, 2011) by demonstrating the importance of accounting for heterogeneity in the underlying social costs of road closures. In the setting of Prague weekdays, we show that using a uniform value of time to determine optimal time incentives will lead cities to mis-price the social cost of closures by up to ninety percent depending on the location and time of road closures. These quantities account for traffic flows along a road segment being composed of many different origins and destinations. However, closures impacting some specific origin-destination pairs impose social costs up to eight times higher than would be predicted by a uniform standard.

Related literature The paper contributes to four strands of literature, which we describe below.

The first strand is the literature on the opportunity cost of time. While the standard labor-leisure choice model implies that an extra hour of leisure should be valued at the shadow wage, the literature starting from the seminal work of Becker (1965) recognizes that an agent's time is also an input to other non-market activities. Recent papers in this literature have used a variety of widely available micro-data to study the trade-off between market goods and time (see, for instance, Aguiar and Hurst (2007); Aguiar et al. (2012); Nevo and Wong (2019)). Though these studies utilize rich and comprehensive datasets of consumption behavior (for example, household scanner data), they are only able to indirectly measure the opportunity cost of time through other market transactions. A related question is how much workers value flexible work schedules. Mas and Pallais (2017) investigate preferences for flexibility in an experiment with call-center workers, Bloom et al. (2015) study work-from-home preferences and performance differences among workers in a large travel agency, and Chen et al. (2017) study the value of work flexibility among Uber drivers. ${ }^{1}$ Our work contributes to this literature in a number of ways. First, we extend the analysis of the opportunity cost of time outside of the workplace and study how this value varies across individuals, space, and time. Second, our data allows us to directly measure consumers' opportunity costs of time

\footnotetext{
${ }^{1}$ The taxi industry has long provided a laboratory for empirical work on flexible work hours. See, e.g., Camerer et al. (1997), Farber (2005), Farber (2008), Crawford and Meng (2011), Thakral and Tô (2017).
} 
and to disaggregate these measures along various dimensions.

The second strand is the emerging literature that utilizes high resolution spatial data to shed light on urban economic activity (Athey et al., 2019; Davis et al., 2017; Couture et al., 2019; Kreindler and Miyauchi, 2019; Almagro and Dominguez-Iino, 2019). ${ }^{2}$ Kreindler and Miyauchi (2019) use individual level commuting flows to estimate a gravity model where consumers choose where to work, as a function of where they live and wage values. Their model yields estimates of the relative desirability of locations. They then show that the implied values from the estimation correlate well with the empirical distribution of wages and night lights in the city. In contrast, since we directly observe how people trade-off waiting time and monetary savings when choosing to move from one location to another, we can obtain a direct measure of their value of spending time at a specific location. Our approach allows us to quantify the intensity of preferences over locations in a way that cannot be accomplished with GPS data alone.

The third strand is the literature in transportation economics and industrial organization, dating to the pioneering work of Daniel McFadden (McFadden, 1974; Domencich and McFadden, 1975), on the value of travel time savings. The studies in this strand measure the benefits of travel time savings through surveys or revealed preference analysis based on mode choice. Our data allow us to directly measure consumers willingness to pay for reductions in waiting time based on choices on the platform. Small (2012) reviews the travel time literature and presents stylized facts suggesting that the value of personal travel time is about $50 \%$ of the gross wage rate, and that the value of travel time increases less than proportionally with income/hourly wage - with elasticity estimates ranging from 0.5 to 0.9 . Couture et al. (2018) study the determinants of driving speed and the deadweight loss of travel, where hours in traffic are valued at half the average wage. Bento et al. (2020) use commuter tollway choices to infer consumers' urgency from their willingness-to-pay for travel time savings. Hall (2018) analyzes the benefits of choice over toll and non-toll lanes. ${ }^{3}$ Finally, a recent literature

\footnotetext{
${ }^{2}$ Although it does not use high resolution spatial data, Su (2018) is also related to this strand. The author studies the causal link between the value of time and gentrification. He argues that the increase in the value of time of high-wage workers led them to seek living areas with shorter commuting times, which then leads to gentrification of former poor, but close to downtown, areas.

${ }^{3}$ There are a host of other studies. For example, the US department of transportation distinguishes in its guide on Valuation of Travel Time between 'on-the-clock' business travelers and personal travel (Belenky, 2011). The department of transportation uses the nationwide median gross compensation based on the 2015 BLS National Occupational Employment Wage Estimates as the value of time in their calculations. For personal travel, estimates are based on survey results from Miller (1989). Like Miller (1989) many studies that estimate time valuations belong to the transportation literature and are largely based on stated preference reports (see Abrantes and Wardman (2011) for the UK and Cirillo and Axhausen (2006) for Germany). Jara-Diaz et al. (2008) combines detailed data (travel diaries and interviews) from Chile, Germany and Switzerland with a theoretical model in the spirit of Becker (1965) to estimate people's value of leisure time. They find that the marginal valuation of leisure is $65.9 \%$ of the average hourly wage in Chile, $119.8 \%$ in Germany and $87.8 \%$ in Switzerland. Borjesson et al. (2012) study two identical surveys given to car commuters in Sweden in 1994 and 2007 and find that people with below median income have elasticity of travel time with respect to income indistinguishable from zero, and those with above median income have elasticity close to 1. Lam and Small (2001) use a survey of California commuters on Route 91, which includes free lanes and tolled "express lanes." The value of time is estimated at $\$ 22.87$, or $72 \%$ of the average wage. Fosgerau et al. (n.d.) study data collected from interviews with over 6,000 Danish people and obtain estimates of the value of time at $67 \%$ of the mean after-tax wage. Significance Quantitative Research (2007) find that the value of time in the Netherlands is about $€ 8.76$, with business trips valued at $€ 24$ per hour. Kreindler (2018) evaluates the
} 
studies equilibrium outcomes resulting from transportation infrastructure. This line of research includes studies that analyze the impact of new roads on driving behavior (Duranton and Turner, 2011), the impact of transit on urban development and spatial sorting (Heblich et al., 2018), the welfare effect of transportation improvements (Allen and Arkolakis, 2019), and the design of optimal transportation networks (Fajgelbaum and Schaal, 2017). Our study complements this literature by showing how transportation options and choices are related to the opportunity cost of time, an important component of the overall welfare of transit infrastructure. We then show how these measures can be used to more accurately set time incentives in a highway procurement setting.

The fourth strand is the literature that studies taxi and ride hail markets. Recent papers analyze the supply side in ride-hail markets. Buchholz (2018) quantifies the impact of uniform pricing regulation and search frictions on the spatial allocation of drivers and passengers in the NYC taxi market; Frechette et al. (2018) assess the effect of entry restrictions and market thickness on efficiency in the NYC taxi market. ${ }^{4}$ In these papers, the demand for taxis is estimated either as a function of prices (Buchholz (2018)) or waiting times (Frechette et al. (2018)). ${ }^{5}$ Cohen et al. (2016) and Castillo (2019) estimate demand for rides on the Uber platform, and in particular Castillo (2019) also estimates the demand for both waiting-time and price. His paper has a different focus and quantifies the benefit of surge pricing, whereas we provide a conceptual framework linking the disutility of waiting-time to the spatial distribution of the value of time. In our setting, we observe a direct and salient choice that includes a price waiting-time trade-off and also a panel-structure for riders, which provides a convincing way to estimate population heterogeneity.

The rest of this paper proceeds as follows. Section 2 describes the institutional setting and our data. Section 3 describes the conceptual framework that motivates our analysis. Section 4 lays out the model of consumer choice and the details of identification and estimation. We present the results of our estimation in Section 5, the application to highway procurement in Section 6 and conclude in Section 7.

\footnotetext{
welfare effect of congestion pricing using both travel behavior data and a field experiment.

${ }^{4}$ In a similar vein, Liu et al. (2019) and Hall et al. (2019) study various aspects of the design of DiDi and Uber, respectively. These papers, however, do not estimate demand. industry.

${ }^{5}$ Brancaccio et al. (2017) and Brancaccio et al. (2020) study closely related models in the context of the oceanic bulk shipping
} 


\section{Setting and Data}

\subsection{A Unique Approach to Matching and Price Discovery}

Liftago is a ride-hail platform, which was founded in 2015 and services rides through licensed taxi drivers. In Prague, all taxis need to be operated by licensed drivers. Moreover, taxis need to be equipped with a physical meter, which captures the number of kilometers traveled in the "occupied" mode and the billed amount. ${ }^{6}$ A licensed driver may find fares by searching for street-hail passengers or by choosing to participate in a dispatch service. Among dispatch options, there are traditional telephone-based dispatch services and, more recently, the app-based ride-hail platform that we study. Note that this regulatory environment is different from most U.S. municipalities, in which there is nearly free entry into the ride-hail market through firms such as Uber and Lyft. ${ }^{7}$

Drivers pay a percentage fee for each ride that is booked through the platform. By tracking both the taxi's GPS and the time of the trip, it provides an approximate fare before the trip begins and a final fare after its completion. While the platform has a strong presence in the Czech Republic, it is less well known in other countries. Because Liftago is not well known internationally few riders are tourists, making it easier to interpret our estimates in light of local economic quantities. ${ }^{8}$

Importantly, drivers and passengers are matched by a combination of a dispatch algorithm and an auction. Whenever a passenger requests a ride, the system looks for nearby available cars and sends requests to a certain number of them, typically four, to elicit an offer. A cab driver who receives a request observes the details of the trip the location of the passenger, the destination, passenger rating and payment via cash or credit. A taxi driver who is interested in performing the job submits a bid that he chooses from a set of pre-programmed tariffs. ${ }^{9}$ A tariff consists of a flag fee, a perminute waiting fee and a per-kilometer fee with a regulatory cap at CZK $36(\approx \$ 1.41)$. The platform takes tariff bids and combines them with a query to Waze, a real-time traffic mapping service and Google subsidiary, which provides estimates of the taxi arrival time (or equivalently the customer waiting time), the trip time and trip distance. The tariff bids are then translated into a single expected price for a trip. The passenger

\footnotetext{
${ }^{6}$ Licensing requires both a fee and an exam. Meters needs to be certified every two years by a state agency. Each meter records the aggregate numbers of kilometers billed together with the revenues.

${ }^{7}$ Uber has also been present in Prague since 2014, but its presence is not as large as in a typical US city of similar size, partially since it is still fighting several legal battles due to various licensing and taxation issues. Since an EU court's decision in December 2017, Uber is viewed as a transportation company and hence its drivers need to be properly licensed.

${ }^{8}$ This is also reflected in the relatively small fraction of airport rides, which comprise about 2 percent of total trips.

${ }^{9}$ When starting the ride, the driver selects a tariff among the options he has pre-programmed on the meter. This is also why the bidding is not completely unrestricted: a typical driver has only about 5 fare combinations on his meter, but there are notable exceptions. Some drivers who specialize in Liftago trips have over 20 different tariffs. Note that neither the passengers nor the other drivers observe tariffs that were not chosen.
} 
then observes bids as final trip prices together with other bid-specific attributes: the waiting time until the taxi arrives, the make and model of the car, and the driver's rating. Importantly, these non-price attributes are automatically attached to each of the bids; in each auction, drivers only have control over the tariff. The passenger may select one of the bids, in which case the ride occurs, or may decline all bids. When the ride is completed, the passenger pays the fare shown on the meter. ${ }^{10}$ Figure 11 shows the interface that riders see on the app before the request and after the request arrives.

A noteworthy consequence of Liftago's mechanism is that it allows for variation in both prices and waiting times: a driver with a high ETA may submit a lower bid than a driver with a low ETA, and vice-versa. Contrast this market clearing mechanism with traditional taxi services, where prices are fixed so that the market clears through adjustments in waiting time, and with other ride-hail platforms, where prices are adjusted to keep waiting times stable.

\subsection{Data}

Our dataset covers 1.9 million trip requests and 1.1 million actual trips on Liftago between September 30, 2016 and June 30, 2018. For each request, we observe the time of the request, the pick-up and drop-off location, trip price bids and estimated waiting times from each driver, and which bid the passenger chose, if any. In addition, we observe a unique identifier for each driver and passenger. There are 1,455 unique drivers and 113,916 unique passengers over the sample period.

For each ride request we complement the data with geospatial and public transportation data from the Google Transit Matrix API, based on the GPS addresses for each origin and destination in the Liftago data. The API data provide us with alternative public transit times for each origin-destination pair. Furthermore, we use hourly rainfall data to attach prevailing weather characteristics to each ride. ${ }^{11}$ Finally, we use data on GPS-specific land values from GIS coded data. ${ }^{12}$

Table 1 summarizes daily activity on the platform. There are on average about 3,000 trip requests each day, of which $61 \%$ become rides. The average bid is $\$ 11.66$ and the average waiting time is 7 minutes. In addition, about one third of the drivers in the sample were active each day. The average number of drivers bidding in each auction is 2.8 , and except in rare cases there are no more than four bids. ${ }^{13}$

\footnotetext{
${ }^{10}$ The app and an email receipt from Liftago only display an estimated amount. However, if the actual amount differs from the estimated amount, customers are encouraged to report the discrepancy. Drivers can be banned from the platform if they are found regularly overcharging.

${ }^{11}$ We obtain this from https: //www . noaa.gov/.

${ }^{12}$ We obtain municipal GIS data from http://www. geoportalpraha.cz.

${ }^{13}$ We discard auctions with more than four bids, representing only $0.33 \%$ of the sample.
} 
Table 1: Bid, Order, and Daily Summary Statistics

\begin{tabular}{lcccc}
\hline Variable & P10 & Mean & P90 & S.D. \\
\hline PANEL A: ORDER SummaRY $(N=1,874,409)$ & & & \\
Price of Trip (USD, across-auction) & 5.65 & 11.66 & 19.75 & 6.261 \\
Wait Time (minutes, across-auction) & 3.00 & 6.8 & 12.00 & 3.816 \\
& & & & \\
PANEL B: BID SUMMARY $(N=5,229,724)$ & & & \\
Number of Bids (within-auction) & 1.00 & 2.79 & 4.00 & 1.087 \\
Price of Trip (USD, within-auction ) & 5.04 & 9.85 & 16.16 & 1.059 \\
Wait Time (minutes, within-auction) & 3.50 & 6.85 & 11.25 & 1.856 \\
& & & & \\
PANEL C: DAILY SuMMARY $(N=638)$ & & & \\
Number of Requests & 1961 & 2938.3 & 4098 & 956.67 \\
Number Rides & 1160 & 1786.2 & 2470 & 557.15 \\
Number Drivers & 411 & 499.23 & 585 & 97.372 \\
\hline
\end{tabular}

NotE: This table shows summary statistics at the bid level (Panel A), the auction level (Panel B) and for entire days (Panel C). P10 refers to the 10th percentile and P90 to the 90th percentile of the respective variable.

Another important feature of the platform is the ability to schedule trips exactly 15 minutes in advance. Scheduled trips represent 7.8 percent of all orders on the platform. While these types of orders do not feature variable waiting times and are not included in our analysis, they suggest that the orders we analyze represent a timely need for trips.

\subsection{Preferences over Time and Money: Intra-daily Patterns}

In this section we provide descriptive evidence for the spatial and inter-temporal patterns in prices, waiting times, and choices. In these dimensions we document large and interpretable heterogeneity in consumer choices, which provides important identifying variation for our model.. Figure 1, panels (a) and (b) show the average trip price and wait times by day of the week and time of the day. Prices are lower during weekday afternoons and higher during weekends, while wait times tend to be substantially higher during the day compared with overnight hours.

Consumers in our data often face a non-trivial trade-off between price and waiting time when choosing among bids. A trade-off implies that there exist options such that one has a lower waiting-time but a higher price and vice-versa. Depending on the time of day about 58-70 percent of auctions involve a trade-off between waiting less and paying more. ${ }^{14}$

Figure 2 shows how consumers solve the trade-off between time and monetary costs

\footnotetext{
${ }^{14}$ Figure 12 in Appendix A shows how the fraction of auctions with a trade-off varies by hour of day.
} 
Figure 1: Prices and Waiting Times by Hour and Day

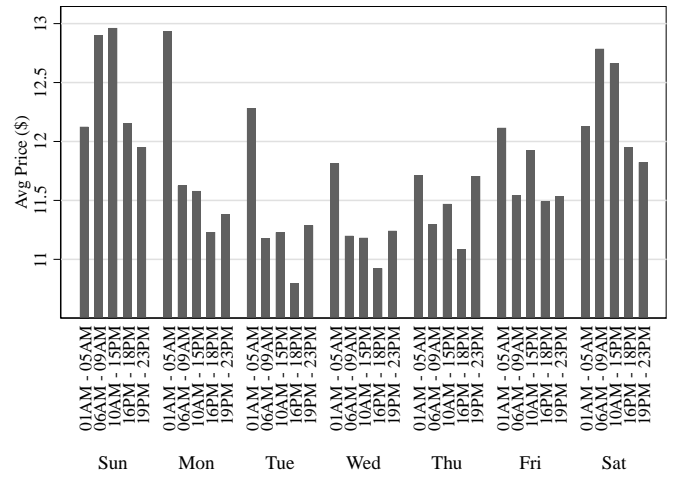

(a) Average Prices

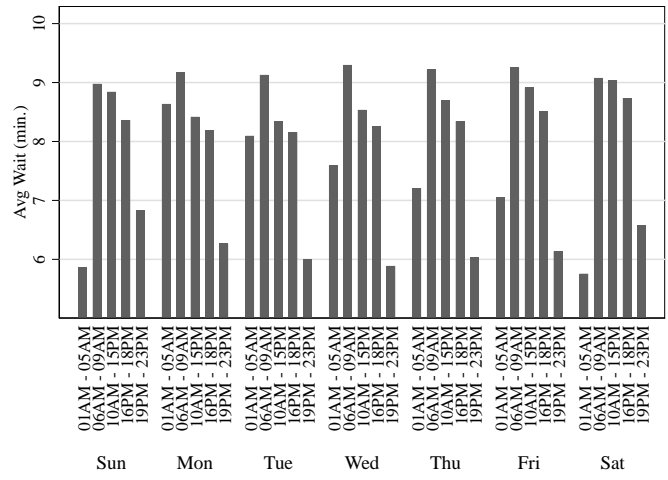

(b) Average Wait Times

at different times of the day. At all times of day, consumers are more likely to pick the minimum price option than the minimum waiting time option. The elasticities that we back out from our model are very much in line with this observation. Moreover, the magnitudes of these differences vary throughout the day. During work hours, there is a a significant dip in the likelihood of choosing the lowest price option and an even larger increase in the likelihood of choosing the lowest wait option. ${ }^{15}$ This pattern can be attributed to some combination of preference heterogeneity across customers as well as within-customer heterogeneity throughout the day. Since we observe customer identifiers, our model leverages the variation across consumers in the timing of trips and the choices within trips.

${ }^{15}$ Note that those two do not have to add up to one since a consumer might, for example, choose a driver with the highest rating even if that driver offers neither the lowest price nor provides the lowest wait time. 
Figure 2: Trade-offs and Choices by Hour

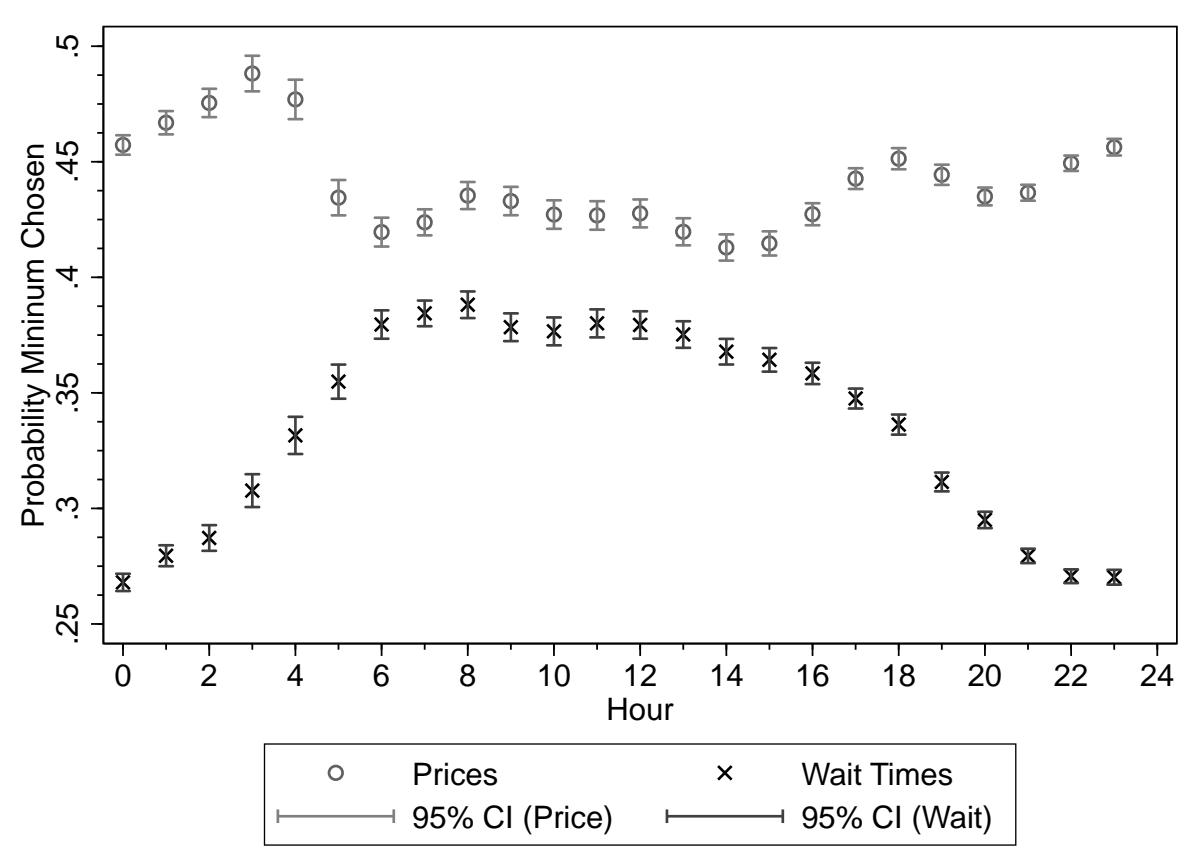

NotE: This graph shows the mean probability that a customer who faces trade-offs between price and waiting time chooses either the lowest price or lowest waiting time.

We next show how choices differ by locations. To partition Prague into smaller regions, we use a data driven approach. We employ a clustering approach using exact GPS locations of trip origins and destinations. The partitioning is done according to a simple $k$-means procedure on latitude and longitude with the number of locations set to $A=30$. We choose this value to balance modeling the richness of spatial preference heterogeneity against sacrifices due to sample size. The resulting map is shown in Figure 18.

Figure 3 compares choices over price and wait times by location. The figure shows, within each pickup location, the probability that customers choose the lowest price and/or wait time among all available bids, computed only within auctions that feature a tradeoff between prices and waiting times. Locations are sorted by the probability of choosing the lowest price. Like Figure 2, Figure 3 demonstrates that consumers exhibit preferences for lower prices and waiting times. It shows that minimum prices are chosen about 2-3 times more often than minimum waiting times, but there is substantial heterogeneity across locations. These differences eventually allow us to infer the different values that riders assign to different locations. We also decompose how much of this variation is coming from place-innate characteristics and how much is driven by differences across people who travel between different locations. We would like to 
Figure 3: Choices and Bids by Pickup Location

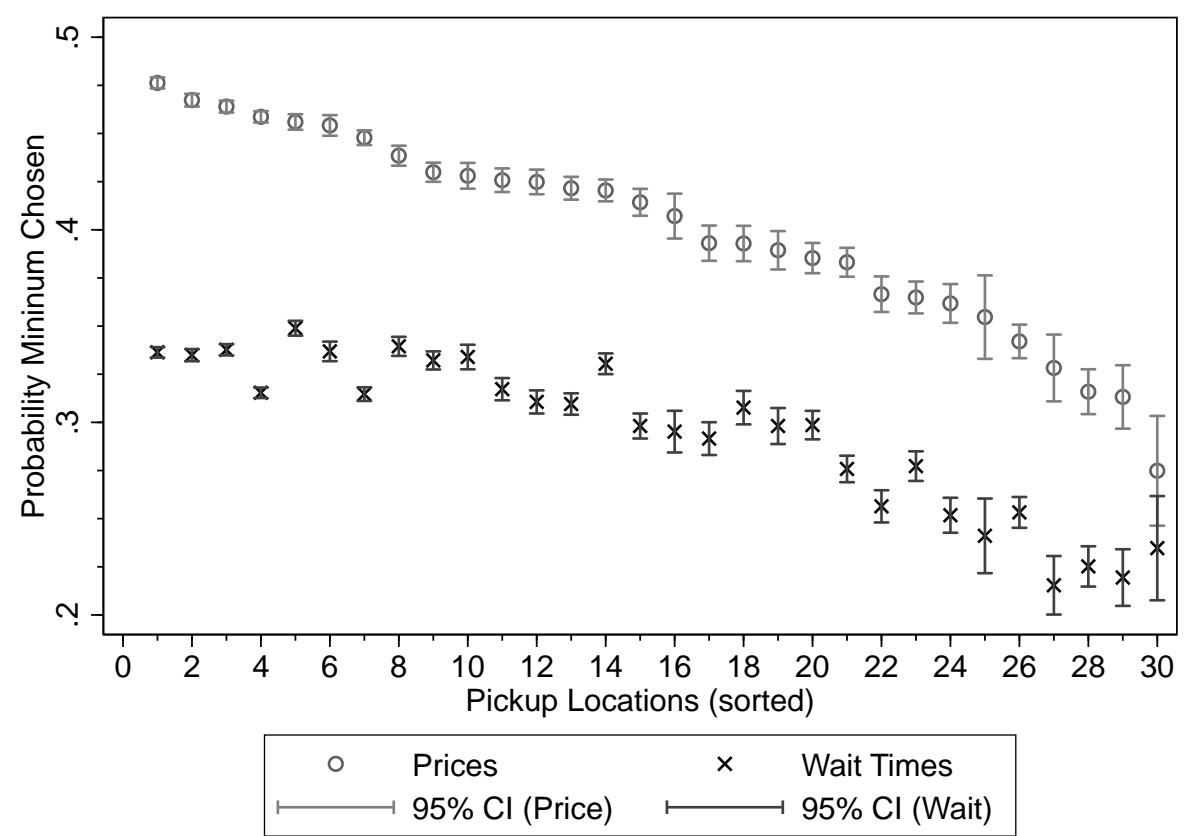

NotE: This figure shows the mean probability that a customer who faces trade-offs between price and waiting time chooses either the lowest price or the lowest waiting time. The locations are sorted by the probability of choosing the lowest price.

emphasize that these differences are computed over the entire sample of trips. Although situational factors might play an important role in passengers' decisions to make a trip, Figure 3 indicates that there are long-run differences in preferences over waiting times across locations.

\section{Conceptual Framework}

We now describe our conceptual framework, which shows how the choices that we observe are related to the underlying value of time at different locations. A consumer's day is characterized by an allocation of time to various activities in different locations. Those activities (e.g., leisure or family time at home versus production at work) have different productivities or different intensities of pleasure at different times. Comparing her options at any given point in time, a consumer thus decides whether to move to a different location and spend her time there. Moving between locations is costly, both in terms of money and time, and a consumer has a choice between various transportation options. Liftago's auction mechanism allows us to observe a particularly clean set of decisions about these transitions.

A consumer is defined by a pair of valuations $\left(\right.$ VOT $^{o}$, vOT $\left.^{d}\right):[0, T] \mapsto \mathbb{R}^{2}$, which 
denote their utility from spending time $t$ at either the origin, $O$, or the destination, $D$. This specification assumes that the value of the time spent on the ride is 0 ; alternatively, this means that vот $^{o}$ represents the value of spending time at the origin, net of the value of the ride, while voT $^{d}$ represents the value of spending time at the destination, net of the value of the ride. Further, these functions will depend on the locations $a \in \mathcal{A}$ of the origin and the destination, which we denote by $\operatorname{voT}_{a}^{o}$, $\operatorname{vOT}_{\hat{a}}^{d}$ if location $a$ is the origin and $\hat{a}$ is the destination.

The consumer starts at time $t=0$ at the origin. However, moving from the origin to the destination involves some travel time and the time waiting for the ride. Once en route, it takes $\Delta$ periods to arrive at the destination. If the consumer asks for a ride at $q \geq 0$ with an estimated time of arrival of $w$ and a price of $p$, her payoff is given by

$$
u(q, w)-p=\int_{0}^{q+w} \operatorname{VOT}^{o}(t) d t+\int_{q+w+\Delta}^{T} \operatorname{VOT}^{d}(t) d t-p .
$$

Figure 4: Origin Destination Trade-Off in Waiting Time Choice

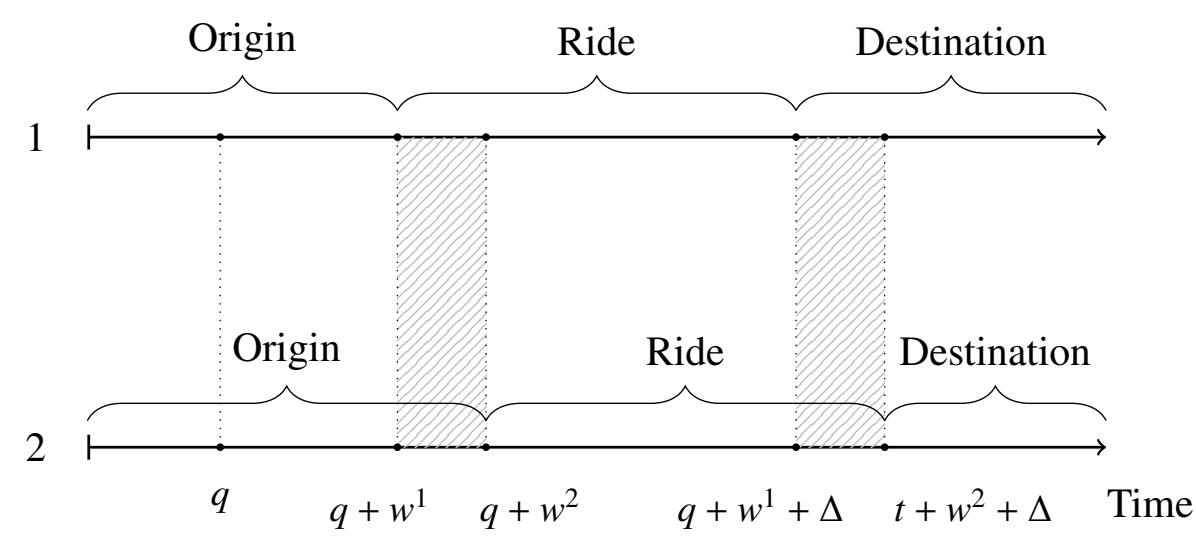

Figure 4 illustrates how the decision to accept a particular bid affects the time allocation between the origin and the destination. Suppose that when consumer requests a ride at time $q$ she receives two bids. Since both drivers are supposed to take the same optimal route, the time from the pickup to the destination is the same and given by $\Delta$. The two bids differ in the estimated time of arrival of the driver. The second bid leads to a longer wait time $w_{2}$. By accepting bid 1, the passenger decides to spend $w_{2}-w_{1}$ less time at the origin and $w_{2}-w_{1}$ more time at the destination.

Let $a$ and $\hat{a}$ denote the origin and destination's locations, respectively. When considering the two trip options, the consumer compares $\operatorname{voT}_{a}^{o} \cdot\left(w_{2}-w_{1}\right)$ against $\operatorname{VOT}_{\hat{a}}^{d} \cdot\left(w_{2}-w_{1}\right)$, up to an approximation. Letting the difference between $w_{2}$ and $w_{1}$ be one unit (for example, one minute), the willingness to pay for waiting time reductions, which we call 
the net value of time, can be expressed as

$$
\operatorname{NVOT}_{a \rightarrow \hat{a}}=\operatorname{VOT}_{\hat{a}}^{d}-\operatorname{VOT}_{a}^{o} .
$$

Different people may assign different values to places, and they may assign different values to the same places at different times. Going forward, the objects that we describe above have $i$ and $t$ subscripts to reflect that we recover a time-of-day and location dependent distribution of the value of time ( $\operatorname{voT}_{\hat{a}, t, i}^{d}$ and $\operatorname{voT}_{a, t, i}^{o}$ ), which gives rise to a distribution of the net value of time ( $\left.\mathrm{NVOT}_{a \rightarrow a}, t, i\right)$. Our data also allows us to incorporate non-linear relationships in waiting time preferences, as a result of unobserved plans or deadlines. For instance, arriving ten minutes late to an appointment could be more than twice as damaging as arriving five minutes late. In Section 4.2 we discuss the interpretation of our model and estimates in the context of these trip-specific qualities.

Since our empirical setting entails choices over trips, we are able to observe distributions of the net value of time but we do not directly observe the underlying value of time. In Appendix $\mathrm{C}$ we formally derive the conditions under which the distributions of $\operatorname{voT}_{a, t, i}^{o}$ and $\operatorname{voT}_{a, t, i}^{d}$ are identified from observed distributions of the net value of time. The result requires one location normalization, either for the origin or the destination. We rely on known deconvolution techniques for non-parametric identification, and for parametric identification (normal distribution) we use a straightforward rank condition.

An important consideration for our measurement is that time at a destination might be more valuable per-se than time at an origin. Such differences might arise from planning and other coordinated activities. For example, people may travel to a particular "destination" location to be productive and then exit the location when their productive task is completed, so that the location becomes an "origin". While we are unable to directly observe plans, our decomposition allows us to estimate the value of time at a location both when it serves as an origin and when it serves as a destination. We are able to do this because we observe riders going in both directions and can thus recover both $\operatorname{NVOT}_{a \rightarrow \hat{a}}$ and $\operatorname{NVOT}_{\hat{a} \rightarrow a}$. With this data we can construct a measure that captures differences in productivity of time use across origin and destination. ${ }^{16}$ To this end, let

\footnotetext{
${ }^{16}$ In the empirical specification we use additional data that is informative about the extent of planning. We obtained the exact location of the customer when a ride was ordered. This allows us to address how much a trip was planned in advance, since we can distinguish, for example, if a customer is standing outside when they place their order and thus has less value for extra time at the origin.
} 
us rewrite the relationship between the NVOT and voT as:

$$
\begin{aligned}
\operatorname{NVOT}_{a \rightarrow \hat{a}}=\operatorname{VOT}_{\hat{a}}^{d}-\operatorname{VOT}_{a}^{o}=\operatorname{VOT}_{\hat{a}}^{d}-\frac{\operatorname{VOT}_{a}^{d}}{\operatorname{VOT}_{a}^{d}} & \cdot \operatorname{VOT}_{a}^{o} \\
& =\operatorname{VOT}_{\hat{a}}^{d}-\delta_{a} \cdot \operatorname{VOT}_{a}^{d}=\operatorname{vOT}_{\hat{a}}-\delta_{a} \cdot \operatorname{VOT}_{a} .
\end{aligned}
$$

This measure is denoted as $\delta_{a}$ and given by the ratio of $\operatorname{voT}_{\hat{a}}^{o}$ to $\operatorname{voT}_{\hat{a}}^{d}$. Following Equation 3, we interpret $\operatorname{voT}_{a}^{d}$ as the inherent location value and $\delta_{a}$ as a depreciation factor due to less (or more) productive time use at the origin. Hereafter we drop superscripts $o$ and $d$.

Finally, when a consumer requests a ride is a choice in and of itself. We discuss in Appendix 4.3 how to interpret our estimates under a model of optimal request times.

\subsection{Model of Travel Choice}

So far, we have treated NVOT $_{a \rightarrow \hat{a}}$ as directly observed. This section describes how to recover NVOT $_{a \rightarrow \hat{a}}$ from a standard discrete choice framework that models indirect utilities over trips. In the next section we describe how to decompose a set of origin-destination NVOTs into location-specific vots.

There is a set of locations $\mathcal{A}=\{1, \ldots, A\}$ indexed by $a$ and a set of consumers $\mathcal{I}=\{1, \ldots, I\}$, indexed by $i$. When presented with a menu of bids (or offers) for a ride between $a$ and $\hat{a}$, the consumer makes a discrete choice between $J$ options. Each alternative $j \in \mathcal{J}=\{1, \ldots, J\}$ is characterized by a tuple consisting of price, wait time, route-characteristics such as distance, characteristics of the car (model, year, color), driver (ratings and name) and a stochastic part $\epsilon_{i, j, t}$. We capture observable trip differences with $\mathbf{x}_{i, j, t}$. We also have to account for an additional term, $\xi_{a, \hat{a}, t}$, that captures unobserved conditions affecting demand on a particular route, such as large sporting events or transit delays. We discuss endogeneity concerns in the estimation section. With this setup, the indirect utility from option $j$ can be written as

$$
u_{i, j, t}=\beta_{i, h_{t}, a, \hat{a}}^{w} \cdot w_{j, t}+\beta_{i, h_{t}}^{p} \cdot p_{j, t}+\beta_{i, h_{t}}^{x} \cdot \mathbf{x}_{i, j, t}+\xi_{a, \hat{a}, t}+\epsilon_{i, j, t}
$$

where $\beta_{i, h_{t}}^{p}$ and $\beta_{i, h_{t}}^{w}$ reflect preferences over waiting time and price; the subscripts $h_{t}, a$, and $\hat{a}$ in $\beta^{w}$. indicate that we allow preferences over waiting time to vary with the hour of the day as well as by origin and destination. Finally, the coefficient $\beta_{i, h_{t}}^{x}$ captures preferences for other bid-specific characteristics (distance, driver's rating, car type, etc.) as well as environmental conditions common to all $j$ : hour-of-day, public transit availability, traffic speeds, trip distance and time, rainfall, origin and destination neighborhoods, 
and whether the order is placed on the street or in a building. Note that the environmental variables allow us to richly condition on many determinants of the outside option.

We can then map the preference parameters in Equation 4 into NVOTs for different locations and different times of day. These NVOT's are obtained via the following equality, which compares the utility of choice $j$ with the utility of some hypothetical option $j^{\prime}$ that adds a single minute to waiting time, but otherwise has the same characteristics. The price difference $p_{j, t}-p_{j^{\prime}, t}$ that solves the equation reflects the additional units of money needed to make consumers, on average, indifferent between paying or waiting more:

$$
\beta_{i, h_{t}}^{p} \cdot p_{j, t}+\beta_{i, h_{t}}^{w} \cdot w_{j, t}=\beta_{i, h_{t}}^{p} \cdot p_{j^{\prime}, t}+\beta_{i, h_{t}}^{w} \cdot\left(w_{j, t}+1\right) .
$$

This implies that a minute of time at destination $\hat{a}$ relative to its value at the origin $a$ is valued as

$$
\operatorname{NVOT}_{a \rightarrow \hat{a}, h_{t}, i}=p_{j, t}-p_{j^{\prime}, t}=\frac{\beta_{i, h_{t}, a, \hat{a}}^{w}}{\beta_{i, h_{t}, a, \hat{a}}^{p}} .
$$

Equation 6 demonstrates that individual estimates of the net value of time can be recovered directly from the estimated demand model by taking a ratio of coefficients.

\section{Estimation}

This section discusses the details of our estimation procedure, which involves two steps. Based on observing the individual choices over bids on the app, we first estimate a likelihood based model. To capture time- and location-specific heterogeneity in time values, we interact all waiting time parameters with time of day fixed effects, and separate fixed effects for each origin and destination. To capture individual-specific heterogeneity, we leverage the panel structure of our data to compute random coefficients on both price and waiting-time using an MCMC procedure. We then use the coefficient estimates from the first step to estimate the NVOT, as we outline above, and a moment-based estimator to separately recover the time and location dependent distribution of the value of time $\left(\operatorname{VoT}_{a, h_{t}, i}\right)$. To allow better interpretation of this heterogeneity, the analysis hereafter utilizes weekday data only.

\subsection{Mixed Logit Discrete Choice Model}

Under the assumption that $\epsilon_{i, j, t}$ are independently and identically distributed according to a Type I extreme value distribution, choosing the maximum among $J$ alternatives with utilities given by Equation 4 reduces to the standard logit. The probability that an 
alternative $j$ will be chosen depends on its relative mean utility and is given by

$$
l\left(w_{j, t}, p_{j, t}, \mathbf{x}_{i, j, t} ; \theta\right)=\frac{\exp \left(\beta_{i, h_{t}, a, \hat{a}}^{w} \cdot w_{j, t}+\beta_{i, h_{t}, a, \hat{a}}^{p} \cdot p_{j, t}+\beta_{i, h_{t}}^{x} \cdot \mathbf{x}_{i, j, t}\right)}{\exp \left(-\xi_{a, \hat{a}, t}\right)+\sum_{j} \exp \left(\beta_{i, h_{t}}^{w} \cdot w_{j, t}+\beta_{i, h_{t}, a, \hat{a}}^{p} \cdot p_{j, t}+\beta_{i, h_{t}}^{x} \cdot \mathbf{x}_{i, j, t}\right)} .
$$

Endogeneity concerns: For bid-specific attributes we as the econometricians are on almost equal footing with consumers because we observe all relevant bid attributes up to the drivers' names and photos. These unobserved features are therefore part of $\epsilon_{i, j, t}$. However, because drivers might condition their bids on $\xi_{a, \hat{a}, t}$, bids could still be correlated with unobservable demand conditions and thereby bias the price coefficients. To deal with this concern, we exploit persistent differences in bids among different drivers. ${ }^{17}$ These differences might, for example, come from pre-programmed bid increments in the meter. However, a straightforward GMM implementation is not feasible since our model relies on individual choice data and is likelihood-based. This prevents us from using standard inversion techniques to isolate $\xi_{a, \hat{a}, t}$ and directly instrument. To get around this we concentrate $\xi_{a, \hat{a}, t}$ out using a control function approach (Petrin and Train, 2010). This step consists of a simple regression of trip prices on a set of driver fixed effects, from which we recover a residual that enters the likelihood as a control. In Figure 13 in Appendix A we show the resulting distribution of fixed effects, which demonstrates that there is large and persistent variation in driver bids. The interquartile range is $\$ 1.90$ or $20 \%$ of the average fare, and the range from the 10 th to the 90 th percentile is $\$ 4.00$ or $43 \%$ of the average fare. Without the control function we find smaller price and waiting time elasticities resulting in an overall mean NVOT that is $19 \%$ lower.

Control variables: We specify $h_{t}$ as follows: the price coefficient is allowed to vary between work hours (defined to be 9am-6pm) and non-work hours. The waiting time coefficient is allowed to vary across five blocks of time. The omitted category is the midnight hour, $12 \mathrm{am}$ to $1 \mathrm{am}$. The remaining blocks are $1 \mathrm{am}$ to 5am, 6am to 9am, 10am to $3 \mathrm{pm}, 4 \mathrm{pm}$ to $6 \mathrm{pm}$, and $7 \mathrm{pm}$ to $12 \mathrm{am}$. We also control for drivers' quality ratings, car type, traffic speed and trip distance. In Equation $4, \beta_{i, h_{t}}^{x}$ captures preferences for other ride- $j$-specific characteristics, which are the distance, the driver's rating, the car type, and environmental conditions common to all $j$.

Outside option: We allow the outside option to vary spatially at the level of each specific order in the data. We control for origin and destination locations, hourly rainfall and whether the trip was ordered from within a building or outside on the street. We also incorporate detailed public transit data including the time-of-day-specific availability of

\footnotetext{
${ }^{17}$ This approach is similar to a literature that exploits different leniency standards of judges, known as the judge design. See, e.g., Waldfogel (1995).
} 
public transit within walking distance between each individual order's origin and destinations points. These characteristics impact the value of the outside option available to consumers, which they earn by choosing no alternative from $\mathcal{J}$. We normalize the value of the outside option to zero during $12 \mathrm{pm}$ and $1 \mathrm{am}$ at a location without any nearby public transit option, when it is not raining and when the order is placed inside a building. Note that this specification allows for spatial (across different origin-destination pairs) and time-of-day variation in the outside option.

Estimation details MCMC: Our estimation exploits the panel structure of our data to capture the full heterogeneity in values of time in a tractable way. In particular, we opt for a hierarchical Bayes mixed-logit model to obtain individual specific estimates for the disutilities of both waiting and money. We use an MCMC method using data augmentation of latent variables as in Tanner and Wong (1987). In this approach the unobserved random coefficients are simulated and then these simulations are treated as data. This method sidesteps the need to evaluate multidimensional integrals by instead sampling from a truncated Normal distribution. Following techniques described in Rossi and Allenby (2003), Rossi et al. (2005) and Train (2009), we now explain the particular version of the Gibbs sampler that we construct.

We assume that the waiting time and price coefficients for each individual are additive in time of day, location, and an individual specific shifter that is normally distributed:

$$
\begin{aligned}
& \beta_{i, h_{t}, a, \hat{a}}^{w}=\beta_{i}^{w}+\beta_{a}^{w}+\beta_{\hat{a}}^{w}+\beta_{h_{t}}, \\
& \beta_{i, h_{t}, a, \hat{a}}^{p}=\beta_{i}^{p}+\beta_{a}^{w}+\beta_{\hat{a}}^{w}+\beta_{h_{t}} .
\end{aligned}
$$

Suppose that $\boldsymbol{\beta}_{i} \sim N(\boldsymbol{\mu}, \boldsymbol{\Sigma})$, where $\boldsymbol{\beta}_{i}=\left(\beta_{i}^{w}, \beta_{i}^{p}\right)$, the vector of coefficients that vary at the individual level. The matrix $\boldsymbol{\Sigma}$ denotes the variance of the individual specific components of the coefficients as well as their covariance. The covariance $\sigma_{w, p}$ tells us whether people who are more elastic to waiting times are also more elastic to price. We would expect this to be true since the utility of income should be related to opportunity costs. However, one can also imagine segments of the population who are wealthy and yet have an abundance of disposable time, such as individuals who may be well-off and retired.

$$
\boldsymbol{\Sigma}=\left(\begin{array}{cc}
\sigma_{w} & \sigma_{w, p} \\
\sigma_{w, p} & \sigma_{p}
\end{array}\right)
$$

We assume that $\boldsymbol{\mu} \sim N\left(\mu_{0}, \Sigma_{0}\right)$, where $\Sigma_{0}$ is a diffuse prior (unboundedly large variance). We assume that the hyper-parameters of the variance are Inverse-Wishart, $\Sigma_{0} \sim I W\left(v_{0}, S_{0}\right)$. One can then iteratively update the $\boldsymbol{\beta}_{i}$-coefficient vector, the mean 
of the coefficients as well as their standard deviations. The specific assumptions we make about the priors lead to conjugate distributions where the posterior mean of $\boldsymbol{\beta}_{i}$ is itself normal and the variance is again in the family of inverse gamma distributions. To describe the updating algorithm, let $\overline{\boldsymbol{\mu}}^{l}$ be the sample mean of coefficients of iteration $l$ in the chain, and let $S^{l}$ be the sample variance of the Inverse-Wishart.

The key simplification exploited in the Gibbs sampler is that one does not have to obtain an analytical expression for the posterior distribution of the $\boldsymbol{\beta}_{i}$ 's, which instead only needs a proportionality factor that can be easily computed at each step. In particular, we have

$$
K\left(\beta_{i} \mid \boldsymbol{\mu}^{l}, \boldsymbol{\Sigma}^{l}, \mathbf{y}_{i}\right) \propto \Pi_{t}^{T_{i}} l\left(y_{t i} ; \boldsymbol{\beta}_{i}\right) \cdot \phi\left(\boldsymbol{\beta}_{i} \mid \boldsymbol{\mu}^{l}, \boldsymbol{\Sigma}^{l}\right),
$$

where $\mathbf{y}_{i}$ is the vector of choices and covariates observed for passenger $i$ with $T_{i}$ observations and $l\left(y_{t i} ; \boldsymbol{\beta}_{i}\right)$ is the likelihood contribution of a particular choice. The iterative updating is then given by the following steps:

1. Draw a new posterior mean $\boldsymbol{\mu}^{l}$ for the distribution of coefficients from $N\left(\overline{\boldsymbol{\mu}}^{l-1}, \frac{W}{N}\right)$.

2. Draw $\boldsymbol{\Sigma}^{l}$ from $I W\left(K+N, S^{l}\right)$, where $S^{l}=\frac{K \cdot I+N \cdot S_{1}}{K+N}$, and $S_{1}=\frac{1}{N} \cdot \sum_{i}^{N}\left(\boldsymbol{\beta}_{i}^{l-1}-\overline{\boldsymbol{\mu}}^{l-1}\right)$. $\left(\boldsymbol{\beta}_{i}^{l-1}-\overline{\boldsymbol{\mu}}^{l-1}\right)^{\prime}$.

3. For each $i$, draw $\boldsymbol{\beta}_{i}^{l}$ according to the Metropolis Hastings algorithm, with new proposal $\boldsymbol{\beta}_{i}^{p l}$ starting from $\boldsymbol{\beta}_{i}^{l-1}$ using density $\phi\left(\boldsymbol{\beta}_{i} \mid \boldsymbol{\mu}^{l}, \boldsymbol{\Sigma}^{l}\right)$.

Such an MCMC procedure is known to be slow for a large dimensional parameter space. To avoid slow convergence we first estimate the model without the random coefficients using standard maximum likelihood and then employ the Gibbs sampler to obtain the distribution of random coefficients separately, starting from the maximum likelihood estimates.

\subsection{Value of Time: Estimation Details}

From the logit model above we obtain the NVOT as the ratio of the price and waiting time coefficients. To decompose the NVOT into the value of time, an additional estimation step is required. Appendix $\mathrm{C}$ provides conditions for identification and shows that we need one location normalization. While our framework allows us to identify the individual-specific vот at arbitrarily fine geographic and temporal resolutions, practical data limitations require us to recover these measures at coarser partitions that aggregate to our locations as defined in Figure 18, at hourly intervals, and in five-percentile bins of individual-specific price versus waiting time preferences, $\beta_{i}^{p} / \beta_{i}^{w}$. We use a moment 
estimator that is based on the simple relationship between the observed $\mathrm{NVOT}_{i, h_{t}, a \rightarrow \hat{a}}$ and the unknown pairs of $\operatorname{VOT}_{i, a, h_{t}}$ and $\delta_{i, a, h_{t}} \cdot \operatorname{vOT}_{i, a, h_{t}}$ :

$$
\operatorname{NVOT}_{i, h_{t}, a \rightarrow \hat{a}}=\operatorname{VOT}_{i, \hat{a}, h_{t}}-\delta_{i, a, h_{t}} \cdot \operatorname{VOT}_{i, a, h_{t}}+\eta_{i, h_{t}, a \rightarrow \hat{a}},
$$

where we interpret $\eta_{i, h_{t}, a \rightarrow \hat{a}}$ as orthogonal measurement error. For each $i$ and $h_{t}$ there are $2 \cdot N_{a}-1$ parameters after normalizing one origin value. For each pair of $\left(i, h_{t}\right)$ we observe $N_{a}^{2}$ equations.

Denoting the collection of vOT's as $\overline{\text { VOT }}$ and requiring that all VOT are positive, we can write our optimization problem as ${ }^{18}$

$$
\begin{array}{ll}
\underset{\overline{\mathrm{VOT}}}{\operatorname{minimize}} & \sum \eta_{i, h_{t}, a \rightarrow \hat{a}}^{2} \\
\text { subject to } & 0 \leq \mathrm{VOT}_{i, a, h_{t}}, \forall i, h_{t}, a \text { and } \delta_{i, 1, h_{1}}=0 \forall i .
\end{array}
$$

We also normalize the origin vor of location one to zero, consistent with the 12am, location one reference category normalization imposed in Equation 4. The normalization is inconsequential because $N_{a}$ is large and the system is over-identified. In fact, we get very similar values for almost all normalizations in which an origin value is set to zero.

\subsection{Optimal Query Times and Interpretation}

Our estimation uses the consumer's choice amongst available trips to estimate the value of time. It does not rely on how these choices came to be; in particular, it does not rely on the query times. Furthermore, in contrast to the model in Small (1982), we have not introduced the notion of an optimal arrival time and have only distinguished between productive time use at the destination and less productive time use at the origin through a simple depreciation factor $\delta$. And yet, given that the decision to move from the origin to the destination reveals that time at the destination has become more valuable than time at the origin, it is natural to ask how our framework which only considers choices over trips connects to the transportation literature, which focuses on optimal scheduling. The rest of this section provides an informal overview of this interpretation; we relegate the formal details to Appendix B.

To illustrate how the consumer chooses when to request a ride, consider the example in Figure 5. In this example, the value of time at the origin is constant, whereas the value at the destination jumps up at $t^{*}$, reflecting that the destination becomes more valuable at $t^{*}$, for instance, due to an upcoming appointment. Assume also that the trip duration from the origin to the destination is $\Delta$. If the consumer queries the app at time

\footnotetext{
${ }^{18}$ To estimate the model we use JuMP (Dunning et al. (2017)).
} 
$q$, she observes at that time the realized waiting times and bids for each of the available rides. For instance, in Figure 5, the consumer has a choice between two trips. Trip 1 has waiting time $w_{1}$, so that the consumer departs at $q+w_{1} \equiv t_{O}^{1}$ and arrives at $q+w_{1}+\Delta \equiv t_{D}^{1}$, whereas trip 2 has a longer waiting time of $w_{2}>w_{1}$. Given the realized waiting times and bids, the consumer chooses the best available ride, the utility of which is evaluated using Equation 1. The consumer chooses the optimal query time before observing the estimated waiting times and bids. Instead, she forms an expectation of the trip choices she will face, and chooses her query time to maximize her expected utility.

Figure 5: Optimal query times and trip choices

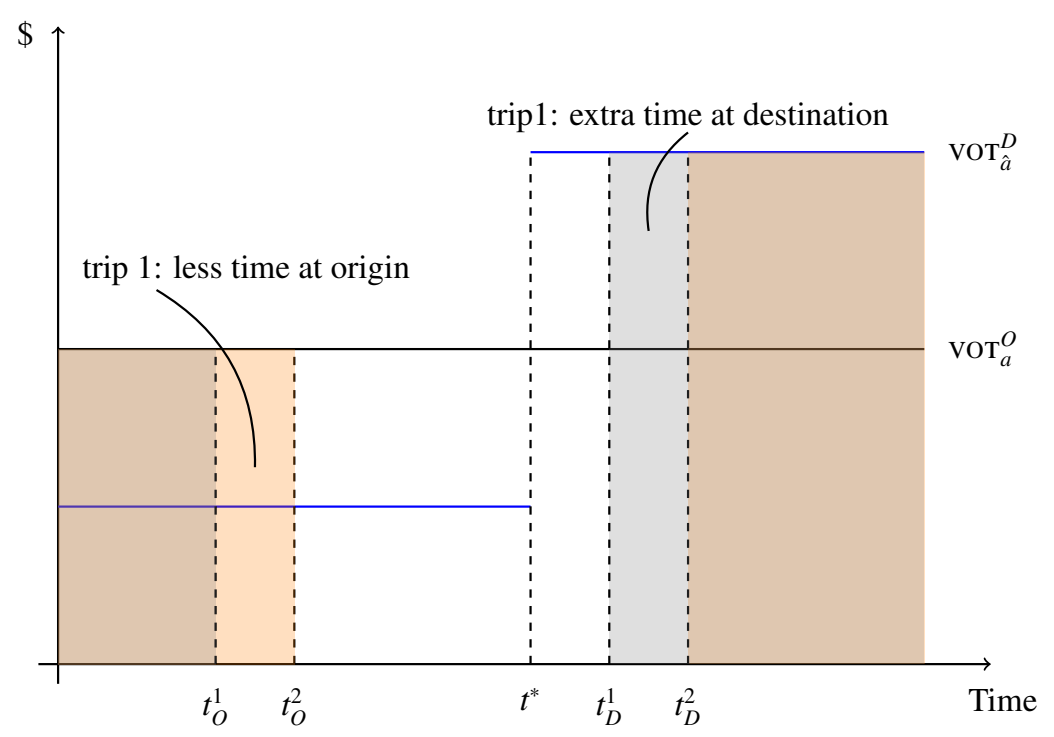

NoтE: This graph shows the differences in value that two trips create. It also shows how the destination value changes after $t^{*}$.

In Appendix B we elaborate on the identification of a model in which query times are optimally chosen. We are able to show that $t^{*}$ is identified under a variety assumptions. Key to this identification is that, even though the consumer optimizes on the time at which the ride is requested, they still face uncertainty over the waiting times and prices they will face. This allows us to infer when a ride would make the consumer late, so that they are willing to pay for shorter ETAs, and when it would make them arrive too early, so that they are willing to pay for longer ETAs. ${ }^{19}$ Furthermore, we show that, even though the observed trip choices reveal preferences about local deviations from the optimal departure time, all parameters of the model are identified under two versions of the model in Equation $1 .^{20}$ The simplest way for observed choices over local deviations

\footnotetext{
${ }^{19}$ Using the language of Equation 1, we can infer when a ETA $w$ is to the left or to the right of the ideal arrival time, $t^{*}$.

${ }^{20}$ In Appendix B we assume that the consumer can finely tune his query time. This is a stylized assumption. However, any friction that prevents the consumer from fully optimizing over the request time would only make our identification argument stronger since it would allow us to measure the consumer's willingness to pay for reductions in waiting times when the value at the origin and the destination are further apart.
} 
to inform us about the value of time more generally is to assume that an individual's underlying values of time at the origin and the destination is constant over some time interval. This assumption would imply that the value expressed over small deviations from the optimal arrival time is linear in time and therefore fully informative about the value of time further away from the optimal arrival time. This is, for instance, what Small (1982) implicitly assumes by letting preferences be linear in the distance to the optimal arrival time. ${ }^{21}$ A look at the data can help us discern whether linearity is a good assumption. Figure 14 in Appendix A shows the likelihood of picking a particular trip as a function of the waiting time, and the likelihood of choosing the inside option as a function of the minimum wait time. While more explicit conditioning on $t^{*}$ is possible, we interpret our distinction between origin- ( $\left.\operatorname{VoT}_{i, a, h_{t}}\right)$ and destination-values $\left(\delta_{i, a, h_{t}} \cdot \operatorname{VOT}_{i, a, h_{t}}\right)$ of time as a reduced form that captures the decline in value attributable to activities being started (at destinations) and concluded (at origins).

\section{Results}

We first present the results from the logit-demand model and the implied elasticities for both waiting time and price. We then present the results on the value of time.

\subsection{Logit Model Results}

Table 2 shows the coefficients and standard errors that we obtain from the demand model. The dis-utility of money is higher than the dis-utility from waiting time, and it is almost identical across working and non-working hours. The intra-daily coefficients on waiting time vary over time-of-day, increasing in absolute magnitude (becoming more negative) into the mid-day peak and declining into the evening. In addition, there is a large amount of origin- and destination-specific heterogeneity in utility. Due to the large number of coefficients, we show the full set of location estimates separately in Figure 20.

The outside option is chosen about $33 \%$ of the time. Several coefficients measure an interaction effect between waiting time and additional factors related to the outside option: public transit availability, whether the trip is ordered on the street, and the presence of rain in the hour the trip was ordered. Since the last two trip-specific features interact with waiting time, their net effects are not immediately apparent. We compute marginal effects to see the impact: rainfall confers a $0.59 \%$ increase in the probability

\footnotetext{
${ }^{21}$ Note that linear preferences in the distance to the optimal arrival time does not necessarily imply that preferences over wait times are linear. Instead, in Small (1982) the consumer exhibits risk aversion over wait times. We illustrate this in Figure 16 in Appendix B.
} 
Table 2: Model Coefficient Estimates

\begin{tabular}{|c|c|c|}
\hline DESCRIPTION & COEFFICIENT & STD ERROR \\
\hline PRICE 6PM-6AM & -1.183 & 0.002 \\
\hline PRICE 6AM-6PM & -1.175 & 0.003 \\
\hline WAItING TIME IAM-5AM & -0.018 & 0.011 \\
\hline Waiting Time 6AM-9AM & -0.081 & 0.011 \\
\hline Waiting TIME IOAM-3PM & -0.089 & 0.01 \\
\hline Waiting TIME 4PM-6PM & -0.062 & 0.01 \\
\hline Waiting Time 7 PM-IIPM & -0.031 & 0.01 \\
\hline WAITING $\times$ ON-STREET ORDER & -0.036 & 0.002 \\
\hline WAITING $\times$ RAINING & 0.015 & 0.004 \\
\hline Waiting Time Squared & -0.006 & 0.0 \\
\hline DRIVER RATING & 11.063 & 0.097 \\
\hline CAR: MID QUaLITY & 0.248 & 0.005 \\
\hline CAR: High Quality & 0.708 & 0.009 \\
\hline TRIP SPEED & -0.051 & 0.002 \\
\hline Alt. Transit Available & 0.044 & 0.007 \\
\hline ORDER ON STREeT & 0.211 & 0.014 \\
\hline RAIN & -0.148 & 0.034 \\
\hline Trip Distance & 5.65 & 0.071 \\
\hline
\end{tabular}

WAITING $\times$ PICKUP LOCATION FE I-30

WAITING $\times$ DROPOFF LOCATION FE I-30

PiCKuP LOCATION FE I-30

Omitted - See Figure 20

DropofF LOCATION FE I-30

HOUR FE

NoTE: This table shows coefficient estimates and standard errors from the logit demand model for each consumer type. The final 120 rows are omitted for exposition. These parameter estimates comprise outside option shifters and waiting time preference interactions with each of 30 pickup and dropoff locations as defined in Section D.1. The omitted results are instead depicted graphically in Figure 20. 
of choosing the outside option. On-street ordering leads to a $0.16 \%$ increase. Within the auction, consumers prefer drivers with higher ratings and better cars. They prefer to use the platform for longer distance trips.

Table 3 shows the elasticities of price and waiting time, computed as the percent change in selecting the bid with respect to a percent change in price and waiting time, respectively.

Table 3: Estimated Elasticities

\begin{tabular}{|c|c|c|c|c|c|}
\hline \multirow{2}{*}{ Time of Day } & \multirow{2}{*}{ Individual Type } & \multicolumn{2}{|c|}{ Bid Level Elasticities } & \multicolumn{2}{|c|}{ Order Level Elasticities } \\
\hline & & Price & Waiting Time & Price & Waiting Time \\
\hline \multirow{5}{*}{$\begin{array}{l}\text { Daytime } \\
\text { 6am-6pm }\end{array}$} & Overall & -4.37 & -1.01 & -3.9 & -0.89 \\
\hline & H Price, H Wait Sensitivity & -8.59 & -1.81 & -7.36 & -1.53 \\
\hline & H Price, L Wait Sensitivity & -2.86 & -0.85 & -2.8 & -0.76 \\
\hline & L Price, H Wait Sensitivity & -5.1 & -1.04 & -4.47 & -0.96 \\
\hline & L Price, L Wait Sensitivity & -2.03 & -0.52 & -2.06 & -0.51 \\
\hline \multirow{5}{*}{$\begin{array}{l}\text { Evening } \\
\text { 6pm-6am }\end{array}$} & Overall & -5.49 & -0.5 & -4.9 & -0.49 \\
\hline & H Price, H Wait Sensitivity & -8.72 & -0.8 & -7.48 & -0.75 \\
\hline & H Price, L Wait Sensitivity & -3.4 & -0.37 & -3.43 & -0.37 \\
\hline & L Price, H Wait Sensitivity & -6.16 & -0.52 & -5.39 & -0.52 \\
\hline & L Price, L Wait Sensitivity & -2.49 & -0.22 & -2.63 & -0.24 \\
\hline
\end{tabular}

Note: This table shows the demand elasticity of price and waiting time across daytime and evening hours and individual type groupings. We distinguish as high $(H)$ price sensitivity individuals who have below median values for $\beta_{i}^{p}$ and low $(L)$ price sensitivity individuals as those with above median values for $\beta_{i}^{p}$, and similarly for waiting time sensitivity. The first two columns show these elasticities among competing bids, reflecting the change in demand due to a $1 \%$ change in price or waiting time on a single bid. The second two columns show the elasticities with respect to choosing the outside option, reflecting a change in demand due to a $1 \%$ change in price or waiting time on all bids.

The table shows a set of bid-level elasticities, which measure the competitiveness of alternative bids, as well as a set of order-level elasticities, which measure the competitiveness of the outside option. We see a general pattern that consumers are much more price elastic than waiting-time elastic: price elasticities range from four to eleven times higher, with starker differences in the evening.

In column 2 we use the estimated joint distribution of random coefficients to categorize four types of individuals as high $(\mathrm{H})$ and low $(\mathrm{L})$ sensitivity to price and waiting time. High price-sensitivity individuals have below median $\beta_{i}^{p}$, meaning they experience the highest disutility from price. Likewise, high waiting-time-sensitivity individuals have below median $\beta_{i}^{w}$. Consumers have highly heterogeneous elasticities: between the two extreme groups, both price and waiting time elasticities differ by about a factor of four. We estimate a modest positive correlation between the sensitivity to price and waiting times: more price-sensitive passengers are also more waiting time sensitive, and vice-versa. 
These elasticity estimates convey that both price and waiting time are important factors in the consumer's decisions, and that waiting time elasticities vary throughout the day in ways that reflect the varying value of work and non-work tasks. ${ }^{22}$

The estimated model fits the data well. In Section E.3, we provide details on how our model fits to both aggregate moments and specific choices.

\subsection{The Net Value of Time}

We now present results for the willingness-to-pay for waiting time reductions implied by our estimates, which we refer to as the net value of time (NVOT), scaled to USD per hour. NVOT represents the difference between the value of time attainable at a destination from the value of time attainable at the trip origin, given the activities and features of each location at each time for each person. We compute the NVOT using the coefficients in Table 2 together with Equation 6, where we account for all trip-specific and environmental factors that affect valuations for the trip and the outside option, such as public transit and rainfall.

Table 4 summarizes the NVOT results. The overall mean NVOT across all trips is $\$ 13.47$ per hour. This means that an average person faced with the option to save five minutes would be willing to pay $\$ 13.47 *(5 / 60)$ or $\$ 1.12$. There are three important sources of heterogeneity underlying this average: individual-, time-of-day- and location-specific heterogeneity. The most prominent source of heterogeneity is between individuals. We again report four groups of individuals, those with above- and belowmedian random coefficient estimates on both price and waiting time preferences. The low price sensitivity and high waiting time sensitivity group exhibits NVOT nearly twice the overall average at $\$ 23.39$ per hour, while individuals with low sensitivity to price and high sensitivity to waiting time have an average NVOT of $\$ 5.00$ per hour. All groups have similar time-of-day patterns, with the highest values in the morning between 6am and 9am. Our value of time estimates are approximately 50\% higher in the late morning and mid-day hours than in the evening and overnight. Finally, we divide Prague's spatial regions into core and non-core, where core regions are the locations including and adjacent to regions 11 and 20 as depicted in Figure 18. In Table 4 we label as urban core trips any trip that involves one of these regions as either origin or destination. We find that the NVOT for trips in core regions is on average $41 \%$ higher than for non-core trips.

\footnotetext{
${ }^{22}$ We can also decompose elasticities by trip origins and destinations as with Table 9 and Table 10. Broadly similar patterns between demand types are revealed, though we see that there are large differences in each elasticity measure from one location to another. In general, price elasticities are more variable than waiting time elasticities.
} 
Table 4: Net Value Of Time Estimates

\begin{tabular}{|c|c|c|c|c|c|c|}
\hline \multirow{2}{*}{ SUBSAMPLE } & \multicolumn{6}{|c|}{ Net Value of Time (NVOT) } \\
\hline & $12 \mathrm{a}-6 \mathrm{a}$ & $6 a-9 a$ & $10 a-2 p$ & $3 p-6 p$ & $7 \mathrm{p}-12 \mathrm{a}$ & All Hours \\
\hline$\overline{\text { AlL TyPES }}$ & 12.56 & 16.38 & 16.63 & 13.91 & 10.96 & 13.47 \\
\hline H Price, H Wait Sensitivity & 14.44 & 17.29 & 17.46 & 15.53 & 13.52 & 15.03 \\
\hline H Price, L Wait Sensitivity & 4.34 & 6.76 & 7.08 & 5.19 & 3.82 & 5.0 \\
\hline L Price, H Wait Sensitivity & 22.51 & 26.21 & 26.44 & 23.96 & 20.01 & 23.39 \\
\hline L Price, L Wait Sensitivity & 8.99 & 12.41 & 12.91 & 9.85 & 2.33 & 10.02 \\
\hline URBAN Core Trips & 12.96 & 17.1 & 17.31 & 14.37 & 11.4 & 13.97 \\
\hline Non-CORE TRIPS & 9.93 & 11.78 & 9.61 & 10.57 & 7.6 & 9.94 \\
\hline
\end{tabular}

NotE: This table shows nVot estimates implied by the logit demand model. All estimates are presented in US dollars.

To interpret our results in finer detail, we note that even a given rider may have a higher or lower NVOT when the circumstances surrounding a ride are more or less urgent. We can therefore view any NVOT estimate that fixes an individual, time-ofday and location to be an average across the circumstances that individual faces. In Section E.6 we demonstrate that our data can be used to recover different NVOT estimates between trips with a drop-off time close to the start of a new hour, which are more likely to involve deadlines, and those at different times. This analysis provides an example of how we might recover different types of trip-specific heterogeneity that is otherwise averaged in the results reported in Table 4.

These results provide insight into the interpretation of geographic (GPS) time-use data, such as cellphone location data. In several studies, authors use this type of data and interpret aggregate location decisions as a measure of place-specific amenities or productive capacity (see, e.g., Kreindler and Miyauchi (2019); Athey et al. (2019)).

Our analysis is, to our knowledge, the first to pair highly granular spatial data, which records where people go within a city, with direct NVOT measures, which capture the relative attractiveness of locations as per our illustration in Figure 4. A natural question is, therefore, how close location values that are derived from GPS tracking are to our direct measures of the relative attractiveness of locations. To answer this question we correlate our WTP for waiting time reductions when going from $a$ to $\hat{a}$ with the fraction of all trips that go from $a$ to $\hat{a}$. The two measures line up well. Figure 6 shows a scatter plot of the nine hundred different directional data points as well as the binscatter points on top. As the plot illustrates, the two measures are highly significantly correlated with a correlation coefficient of 0.56 and a $t$-statistic of 20.46. This correlation is highest during the middle of the day and lowest at midnight. 
These results convey that travel flows appear to be a good but not perfect measure of time valuations. The variation in the correlation between our voT measure and travel flows throughout the day gives further guidance on when travel flows align better with place-specific values of time. When available, value of time estimates based on revealed preferences in transportation markets provide a sharper quantification of the relative attractiveness of locations than the use of travel flows alone. In the next section, we explore this idea further by estimating the model outlined in Section 3, which microfounds the expressed willingness to pay to reduce waiting times through place-specific values of time.

Figure 6: Relationship between Travel Flows and the NVOT

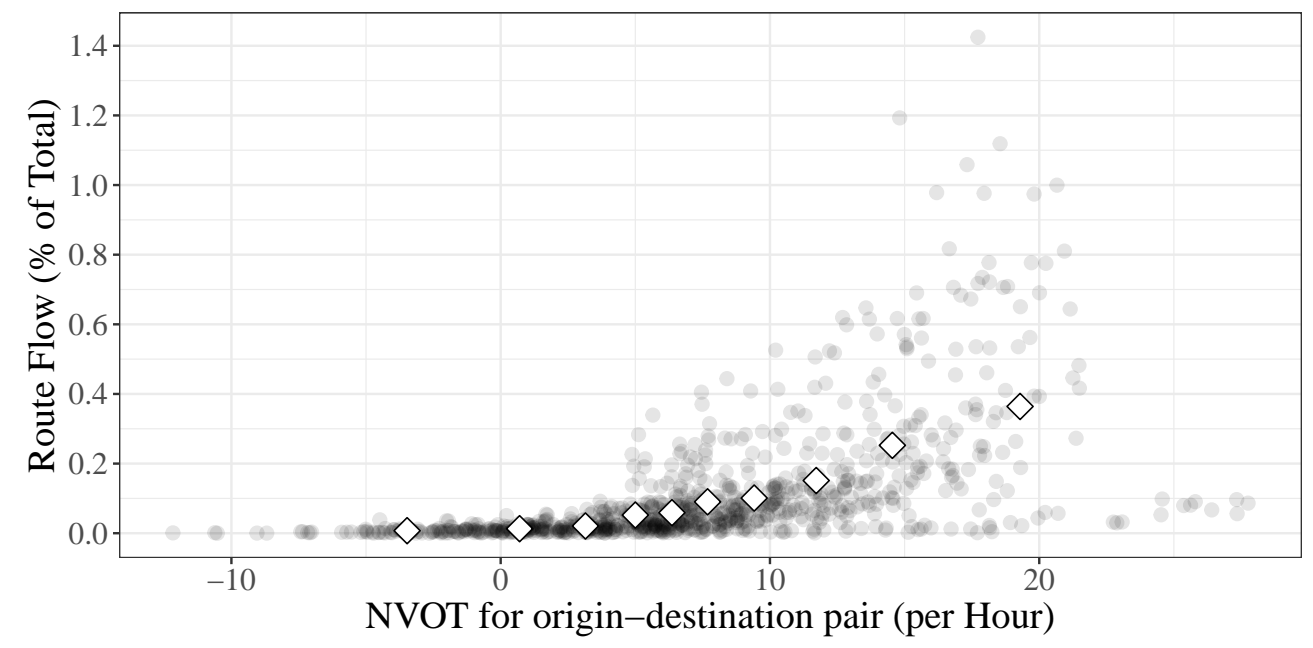

Note: This graph shows the scatter (transparent round dots) and binscatter (white diamonds) relationship between the NVOT for an origin-destination pair and the respective traffic flow (as a fraction of the total).

\subsection{The Value of Time}

Equation 2 expresses how a transportation-specific measure - the willingness to pay for waiting time reductions, or NVOT - is related to the value of time. A measure of the value of time (VOT) is useful outside the context of transportation and can be seen as a summary measure of the value that a person expresses for the set of activities at a place and time of day. In this section we highlight several stylized facts about the distribution of voT across places, time, and individuals.

Figure 7 shows the unconditional distribution of the value of time (USD/hour) that we back out. The histogram reveals large variation. The interquartile range goes from $\$ 6.80$ per hour to $\$ 16.30$ per hour. The tenth percentile of the distribution is $\$ 3.70$ per hour and the ninetieth percentile is $\$ 21.30$ per hour. 
Figure 7: Histogram of voT

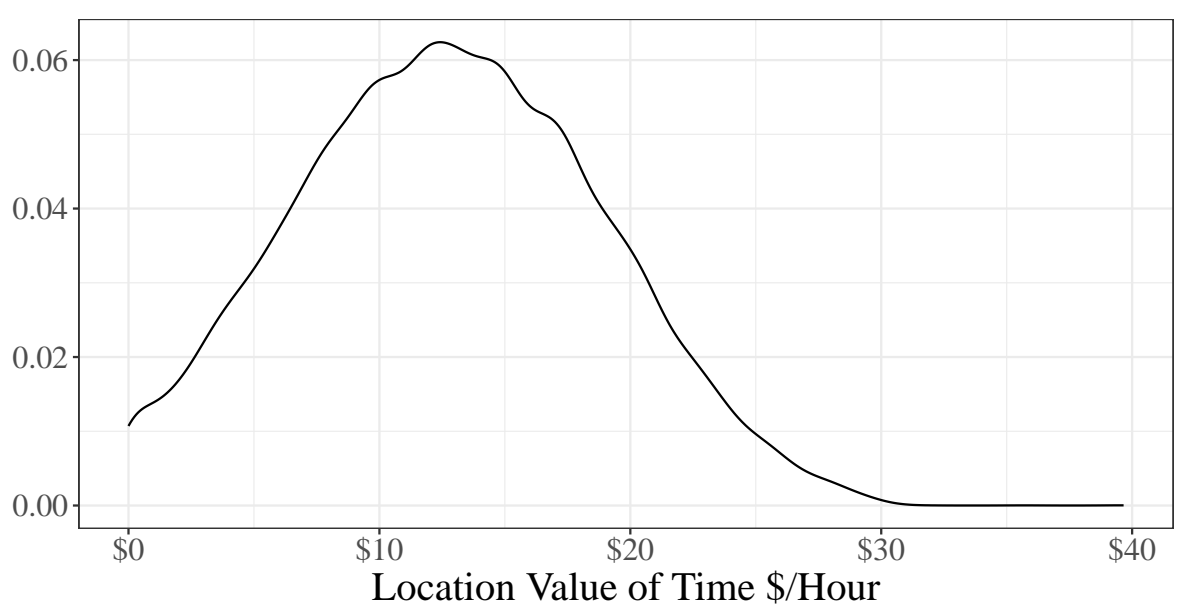

NotE: This graph shows the unconditional distribution of the values of time that we back out.

Spatial Heterogeneity Figure 8 shows vот heterogeneity by location, measured as the average across people and time of day of all vor estimates for this location. Going from the lowest-value location to the highest-value location implies a difference of $\$ 10.83$ in the hourly value of time, or an increase of $51.96 \%$. However, the interquartile range is already substantially smaller with a difference of $\$ 2.89$, or $17.78 \%$. This relatively flat distribution suggests that location heterogeneity is not the main driver of variation in the value of time.

These results offer a direct measure of the short-run monetary value that people assign to spending time at different places. This distribution is different from other measures studied in the literature. For example, economists have devoted considerable attention to measuring the long run effect of place of residence on individual outcomes (Chetty et al., 2018; Couture et al., 2019). However, place of residence might be an imperfect measure of the extent to which people benefit from the resources that different places have to offer. For instance, a study of "experienced segregation" based on GPS data reveals that time use across different spatial regions is less segregated than residential locations (Athey et al., 2019). Another example is Davis et al. (2017) who use Yelp data to show that restaurant consumption is only half as segregated as residences. Our vot measure encompasses the valuations stemming from the full set of activities of individuals in our sample.

To explore how our value of time measures compare to other quantities that capture spatial differences in economic activity, we correlate our voT estimates with real estate values. We use a binscatter plot to show this relationship in Figure 9. There is a strong positive relationship between real estate prices and our voT measures. However, the 
Figure 8: Map of voт Estimates in Prague

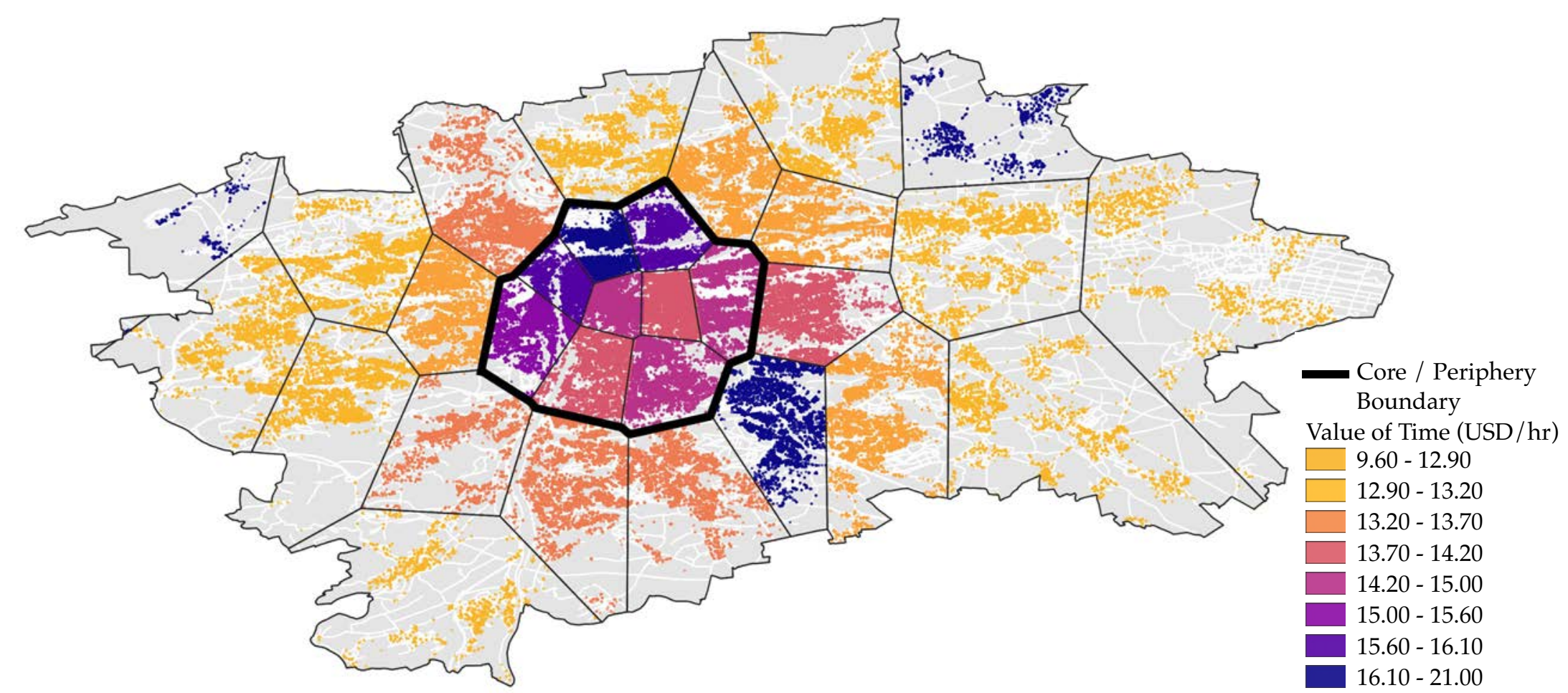

Note: This figure depicts each GPS destination point in the Liftago data shaded by the average value of time in that destination, or $\operatorname{vot}_{a}^{d}$ across places $a \in\{1, \ldots, 30\}$ within the city limits of Prague. White lines depict the city's street map. The bold black line delineates the boundaries that we specify as the urban core. 
"returns" to higher land values are diminishing. From a regression of log-vOT on log land-values we measure an elasticity of $0.25(p<0.001)$. So for every $1 \%$ higher land value we measure a value of time that is $0.25 \%$ higher. On the one hand, this strong positive relationship validates our measures since we have not used land values at any point in the estimation. On the other hand, we find that with an $R^{2}$ of less than 0.02 , real estate prices can only explain a very small percentage of the variation in vот. This finding highlights how our value of time measures serve as a unique short-run analog to other widely available long-run measures of economic value across space. Such measures are important when policies target short-run decision making. We illustrate this in our application on procurement incentives.

Figure 9: Location-specific voT and Land Values

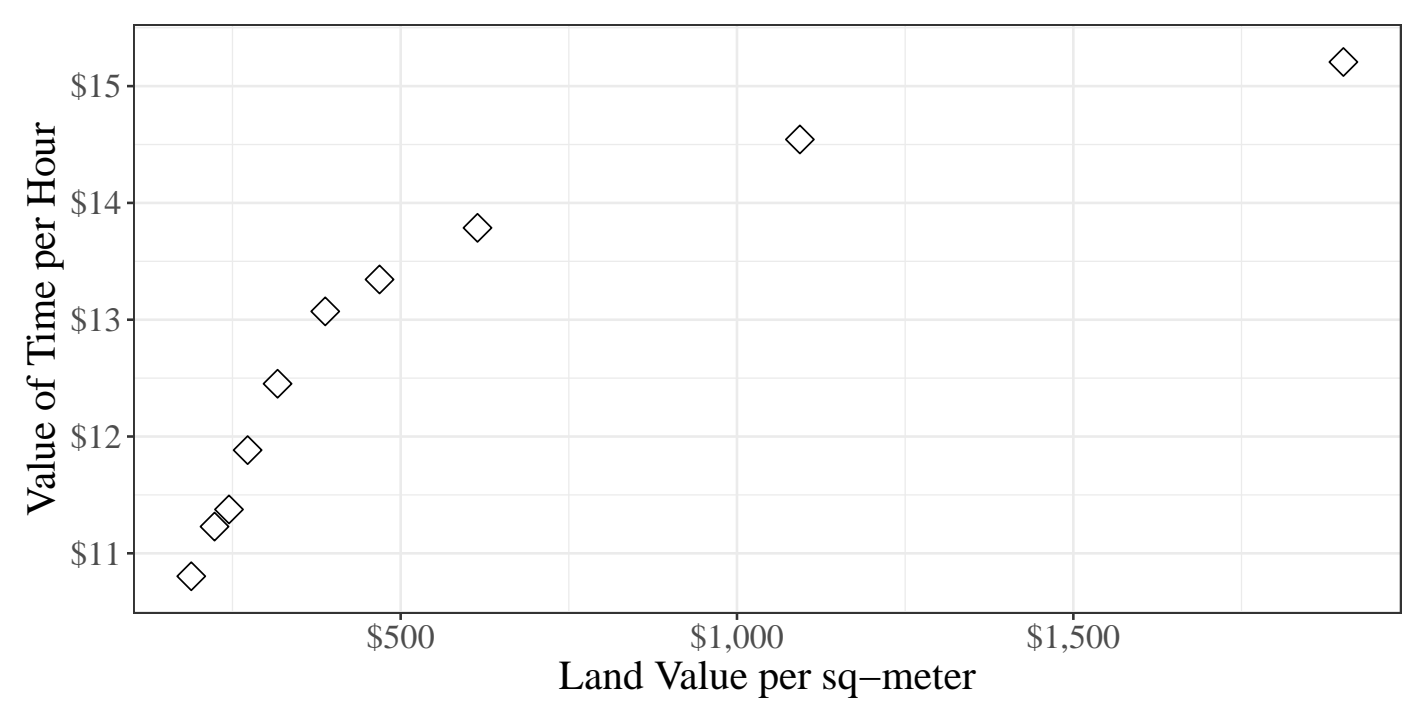

Note: This figure presents a binscatter plot of the value of time in each location compared to the average land values within each location. The $\mathrm{x}$-axis orders the thirty locations by their average land value. The $\mathrm{y}$-axis shows the average value of time in each of these locations.

Time-of-day and Person Heterogeneity For clarity of exposition, we group time of day into two categories - work-hours, defined as the hours $6 \mathrm{am}-6 \mathrm{pm}$, and non-workhours. Table 5 gives an overview of means and standard deviations of the voT estimates across these times of day for the four different groups of passengers. During both work and non-work hours, the (L Price, H Wait) group expresses a vor that is almost three times that of the (H Price, L Wait) group. Within type, however, the difference between work-hours and non-work-hours is modest. Across all groups the ratio of non-workhours to work-hours vOT ranges from $74 \%$ to $87 \%$. Those ratios are ordered in terms of the average vот of these groups with (L Price, $\mathrm{H}$ Wait) types having the highest and $(\mathrm{H}$ Price, L Wait) types the lowest. 
Table 5: Summary Statistics of Value of Time

\begin{tabular}{lccccc}
\hline & \multicolumn{2}{c}{ Work Time (USD) } & \multicolumn{2}{c}{ Non Work Time (USD) } & $\begin{array}{c}\text { Non Work Time vot / } \\
\text { Work Time vot (\%) }\end{array}$ \\
& MeAn & STD & MeAN & STD & \\
\hline Location Values $\left(v_{i, a, h_{t}}\right)$ & & & & & 0.82 \\
All & 17.15 & 10.29 & 14.02 & 10.39 & 0.83 \\
H Price, H Wait Sensitivity & 19.18 & 7.0 & 15.95 & 7.05 & 0.74 \\
H Price, L Wait Sensitivity & 9.79 & 4.82 & 7.25 & 6.24 & 0.87 \\
L Price, H Wait Sensitivity & 27.05 & 12.43 & 23.45 & 12.73 & 0.78 \\
L Price, L Wait Sensitivity & 12.66 & 4.64 & 9.83 & 5.85 & \\
\hline
\end{tabular}

Note: This table shows summary statistics on the estimated value of time overall and across four groups of individuals. We separate estimates by the work hours from $6 \mathrm{am}-6 \mathrm{pm}$ and non work hours from $6 \mathrm{pm}-6 \mathrm{am}$. The last column shows value of non-work time as a percentage of the value of work time.

\subsubsection{Variance Decomposition}

Our estimates also allow us to study whether different strata of society value different places differently. We exploit the fact that our data contain a panel of riders, so we can observe the same rider making decisions about time allocations across many different places. If most of the variation in the value of time is driven by places and not people, this would suggest that place-specific factors provide equal benefit to different people. For example, there might be differences in the provision of public goods across places, which are equally available to all people. On the other hand, differences in the value of time in different places might be predominantly driven by differences across people. This would be true, for example, if people with high value of time derive utility from places solely because their workplaces are there, and otherwise derive no amenity value from public services.

We perform a variance decomposition to separate these two sources of variation and investigate their relationship in more detail. A variance decomposition also allows us to investigate whether people with high average vот spend more time in places with high average vOT. ${ }^{23}$ The measurement of such sorting effects has been of long standing interest in the economics of marriage markets (Becker (1973)), labor markets, (Abowd et al., 1999; Eeckhout and Kircher, 2018; Shimer and Smith, 2000) and, more recently, housing markets (Couture et al. (2019)). Our approach is similar to the strand of labor economics literature that decomposes wages into firm specific components and worker specific components, most importantly Abowd et al. (1999). For the variance decom-

\footnotetext{
${ }^{23}$ The location specific estimates should not necessarily be interpreted as deep structural parameters that are inherent to a location. They could capture certain agglomeration effects, such as the taste for meeting certain people who typically can be found at a given location.
} 
position we derive an accounting equation from the following regression model:

$$
\operatorname{VOT}_{i, a, h_{t}}=\alpha_{i}+\eta_{h_{t}}+\gamma_{a}+\epsilon_{i, t}
$$

where $\alpha_{i}$ is a person fixed effect, $\eta_{h_{t}}$ is a time-period fixed effect, $\gamma_{a}$ is a location fixed effect, and $\epsilon_{i, t}$ is a residual. Due to the curse of dimensionality, we run this specification by replacing individual values with percentile means, so $i$ becomes an indicator of the percentile bin. ${ }^{24}$ This regression then gives rise to the following variance decomposition:

$$
\begin{aligned}
& \operatorname{Var}(\mathrm{vOT})=\operatorname{Var}(\alpha)+\operatorname{Var}(\eta)+\operatorname{Var}(\gamma)+ \\
& 2 \cdot \operatorname{Cov}(\alpha, \eta)+2 \cdot \operatorname{Cov}(\alpha, \gamma)+2 \cdot \operatorname{Cov}(\gamma, \eta)+\operatorname{Var}(\epsilon) .
\end{aligned}
$$

Table 6 shows the results from this exercise. At 78\%, by far the largest contributor to the observed variance of the voT are differences across people. In comparison, locationspecific variance is small and accounts for $10 \%$ of the total. Intra-daily changes in the

\begin{tabular}{|c|c|c|c|c|c|c|}
\hline $\operatorname{Var}\left(\alpha_{i}\right)$ & $\operatorname{Var}\left(\eta_{h_{t}}\right)$ & $\operatorname{Var}\left(\gamma_{a}\right)$ & $2 \cdot \operatorname{Cov}\left(\alpha_{i}, \gamma_{a}\right)$ & $2 \cdot \operatorname{Cov}\left(\alpha_{i}, \gamma_{h_{t}}\right)$ & $2 \cdot \operatorname{Cov}\left(\gamma_{a}, \eta_{h_{t}}\right)$ & $\operatorname{Var}\left(\epsilon_{i, t}\right)$ \\
\hline 27.7 & 3.25 & 3.5 & -0.4 & -0.1 & 0.3 & 1.6 \\
\hline 0.78 & 0.09 & 0.1 & -0.01 & -0.002 & 0.008 & 0.04 \\
\hline
\end{tabular}
vOT contribute another $9 \%$.

Table 6: Variance Decomposition of the Value of Time

Note: The first row of this table shows the variances of each of the components of the model in Equation 13. The second row shows the variance of each of the components divided by the total variance, which can loosely be interpreted as fractions of the total variance.

Moreover, the covariances show that high voT people do not spend more time in high VOT locations. With a covariance of -0.4 , we observe a slightly negative sorting effect. Similarly, there is a negative correlation between people who express a high value of time and the times of day with higher average vот. ${ }^{25}$

\subsubsection{Discrepancy between Value of Time at Origin and Destination}

We next discuss our estimates of differentially effective time use at origin and destination, as measured by $\delta_{i, a, h_{t}}$. Table 7 gives an overview. Across the entire population,

\footnotetext{
${ }^{24}$ Our panel is not long enough to observe a given individual in all possible combinations of origin, destination and time of day.

${ }^{25}$ To address the concern that our results could be driven by specific aspects of the decomposition, we have repeated this same exercise with the NVOT as opposed to VOT. Location fixed effects in this exercise are defined over directional pairs since the net value of time is defined over those pairs. Under this alternative exercise, we come to a very similar conclusion. Most of the variation is driven by differences across people instead of differences across places.
} 
unplanned time at the origin is only $31 \%$ as valuable as time at the destination during work hours, while it is $36 \%$ as valuable during non-work hours. Holding the location fixed, meetings and other types of work-required planning therefore lead to a larger discrepancy in the value of planned and unplanned time. Moreover, there is a large difference in $\delta$ within the population. Not surprisingly, people that are more waiting time sensitive (H Wait Sensitivity) have a larger discrepancy in the value of planned and unplanned time. This shows that higher willingness to pay for lower waiting times is driven both by a larger inherent value of time at any given location and also a bigger discrepancy between the value of planned and unplanned time.

Table 7: Summary Statistics for $\delta_{i, a, h_{t}}$

\begin{tabular}{lcccc}
\hline & \multicolumn{3}{c}{ Work Time } & \multicolumn{2}{c}{ Non Work Time } \\
& MEAN & STD & MEAN & STD \\
\hline Origin Depreciation Factors $\left(\delta_{i, a, h_{t}}\right)$ in USD & & & & \\
All & 0.18 & 0.17 & 0.2 & 0.24 \\
H Price, H Wait Sensitivity & 0.14 & 0.11 & 0.12 & 0.11 \\
H Price, L Wait Sensitivity & 0.32 & 0.34 & 0.5 & 0.79 \\
L Price, H Wait Sensitivity & 0.14 & 0.12 & 0.14 & 0.12 \\
L Price, L Wait Sensitivity & 0.18 & 0.18 & 0.21 & 0.25 \\
\hline
\end{tabular}

NotE: This table shows summary statistics for our estimates that capture how much less productive time at the origin is relative to the destination.

To summarize our results, we find large differences in the value of time across individuals and also large differences in differentially productive time use across locations. Most of the variation in the value of time comes from person-specific heterogeneity as opposed to location-specific heterogeneity. Interestingly, these results suggest that in the short run, different places do not confer benefits that are equally valued by everyone. These results are also an important short-run complement to the recent debate around the importance of place-specific factors for long-run outcomes. Another interesting implication lies in the finding that the value of time for high types can, in part, be explained by a greater need for planning as measured by the origin depreciation factor $\delta$. This suggests that high vот types depend to a larger extent on complementary inputs that require coordination. For example, high skilled work places might require more meetings and coordination with others to use time productively. 


\section{Application: Highway Procurement}

The value of time and its heterogeneity impacts many markets in a variety of ways, but nowhere is its impact more direct than in the transportation sector, as a central goal of roads and transit infrastructure is to generate time savings.

In this section, we consider the problem of using time-incentives in a highway procurement setting. By design, repairing and maintaining highways entails closures which slow down traffic and may induce substantial congestion. Some state and local authorities use time-incentives as part of procurement procedures, which enable contractors to earn higher payments for faster completion or require them to pay fines for delays. This problem was introduced to the economics literature by Lewis and Bajari (2011), where the authors examine CalTrans data to study the welfare gains associated with procurement auctions that incorporate bidding on both time to completion and price into a scoring auction framework. The authors find that such procurement mechanisms used by CalTrans raised social welfare by $30 \%$ of project costs by generating time savings despite higher project prices.

To quantify the social cost of traffic delays due to construction, the authors apply a uniform $\$ 12$ per hour value of time. This value is derived from engineering estimates and accounts for the possibility of multiple passengers in the same car. ${ }^{26}$ Since our approach allows us to recover economic estimates of time valuation, we re-consider the problem of highway procurement and calculating the social costs of road work. Beyond quantifying average time costs, we use our estimates to further explore how optimal time-incentives are impacted by underlying heterogeneity in the value of time. By examining the impact of road closures in different areas of the city and, potentially, at only certain times of day, we show that the social cost of these closures can vary considerably. This result underscores the importance of incorporating value of time heterogeneity into optimal time incentives.

As in Lewis and Bajari (2011), the social cost of a delay associated with a project $j$ can be written as

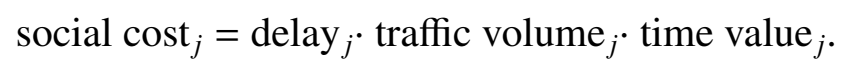

Repair projects cause delays due to reduced travel speeds. The delay depends on the nature of road repairs, for example the number of lanes that are closed and the length of the closure. Although road work often creates delays by lowering speed limits

\footnotetext{
${ }^{26} \mathrm{CalTrans}$ does not specify the source of the underlying calculation. Updated estimates from the same source now put this value at $\$ 13$ per hour. See https://dot.ca.gov/-/media/dot-media/programs/maintenance/documents/ office-of-concrete-pavement/life-cycle-cost-analysis/value-of-user-time-2013-a11y.pdf.
} 
and reducing lanes, the realized traffic volumes and speeds should still be regarded as equilibrium quantities; some travelers will re-optimize plans and activities in the face of delays. Since we do not observe specific roadways or construction projects, we follow Lewis and Bajari (2011) in considering a hypothetical setting where the equilibrium impact of a closure results in three minutes of added delay, which is roughly equivalent to a decline in speed from $55 \mathrm{mph}$ to $35 \mathrm{mph}$ for five miles.

To map delays to time costs, we first use our vот measures by origin and destination. When delays are unanticipated, travelers will not plan ahead, leading to a late arrival at the cost of time at the destination, or vot. Conversely, anticipated delays will lead to early departure, at the cost of time at the origin, i.e., $\delta \cdot$ voT. By examining overall averages we derive that anticipated delay costs to travelers on our platform at 9am are equal to $\$ 0.15$ per trip while unanticipated costs are equal to $\$ 0.76$ per trip. At $9 \mathrm{pm}$, however, these costs drop to $\$ 0.09$ and $\$ 0.54$. Underlying these averages, there are important spatial differences. On some routes, the average cost per trip is as high as $\$ 2.50$. How much construction delays are anticipated by drivers will vary according to several factors, such as how long the delays are in effect, how many drivers are repeated users of the road, and how many check traffic conditions before driving. Hereafter we assume that half of drivers face expected congestion and half do not. This split is motivated by the proportion of trips during an average weekday in Prague that occur during the commuting times of $6 a-10 a$ and $2 p-7 p$, which are 51 percent of total traffic volume.

To extrapolate from our setting to the population of Prague drivers, we need to address the selection of users in our platform. The population within Prague from which our estimates are drawn (i.e., taxi passengers) is likely to be selected on high-VOT individuals compared with the average Prague commuter. In order to extrapolate from our hourly congestion cost estimates, we first adjust these costs to reflect the opportunity cost of car commuters who are not on the Liftago platform.

We take advantage of the fact that the value of time that we measure at 9am is very close to the wage rate of those individuals as measured by a small survey that we conducted among passengers together with Liftago. We find that the mean wage rate among survey participants is $\$ 15.23$, very close to our measurement of the vot during work hours, which is $\$ 15.44$. This time period also most plausibly captures the value assigned to work related activities. We therefore assume that at 9:00am, a typical workday starting time, our vот estimate is equal to users' average wage. The mean wage among Prague residents, however, is $\$ 9.15$ per-hour. We specify an adjustment factor $\alpha=9.15 / 15.44=0.59$ and scale all voT results by $\alpha$. Combining these assumptions allows us to infer, for example, that at $5 \mathrm{pm}$ when Liftago riders face an unanticipated 
congestion cost of $\$ 1.50$ per trip, the average car commuter would face a cost of $\$ 0.89$ per trip. Finally, we also scale by the average occupancy rate of cars in Prague, which is 1.3 as reported by the 2018 Prague Transportation Yearbook. The final scaling factor is therefore $0.59 \cdot 1.23=0.767$. The average scaled per-trip delay costs due to lost origin and destination vOT are $\$ 0.098$ and $\$ 0.51$, respectively.

Table 8: Estimated Per-Trip Closure Costs by Time of Day

\begin{tabular}{|c|c|c|c|c|c|c|c|c|}
\hline & \multicolumn{8}{|c|}{ Time-of-Day } \\
\hline & 3:00am & 6:00am & 9:00am & 12:00pm & 3:00pm & 6:00pm & 9:00pm & 12:00am \\
\hline \multicolumn{9}{|l|}{ A. Uniform Cost Baseline } \\
\hline Uniform Price & $\$ 0.30$ & $\$ 0.30$ & $\$ 0.30$ & $\$ 0.30$ & $\$ 0.30$ & $\$ 0.30$ & $\$ 0.30$ & $\$ 0.30$ \\
\hline \multicolumn{9}{|c|}{ B. All Routes with Time Variation } \\
\hline All Routes & $\$ 0.31$ & $\$ 0.29$ & $\$ 0.36$ & $\$ 0.36$ & $\$ 0.37$ & $\$ 0.34$ & $\$ 0.27$ & $\$ 0.24$ \\
\hline$\%$ change & 0.02 & -0.05 & 0.17 & 0.19 & 0.21 & 0.12 & -0.1 & -0.2 \\
\hline All, Volume Weighted & $\$ 0.05$ & $\$ 0.06$ & $\$ 0.51$ & $\$ 0.52$ & $\$ 0.54$ & $\$ 0.56$ & $\$ 0.33$ & $\$ 0.12$ \\
\hline$\%$ change & -0.83 & -0.8 & 0.68 & 0.71 & 0.77 & 0.85 & 0.08 & -0.6 \\
\hline \multicolumn{9}{|c|}{ C. Routes by Destination and Time } \\
\hline Highest-VOT Destination & $\$ 0.26$ & $\$ 0.31$ & $\$ 0.42$ & $\$ 0.35$ & $\$ 0.35$ & $\$ 0.39$ & $\$ 0.36$ & $\$ 0.26$ \\
\hline$\%$ change & -0.13 & 0.01 & 0.38 & 0.16 & 0.15 & 0.28 & 0.19 & -0.14 \\
\hline Median-VOT Destination & $\$ 0.20$ & $\$ 0.20$ & $\$ 0.26$ & $\$ 0.27$ & $\$ 0.30$ & $\$ 0.32$ & $\$ 0.27$ & $\$ 0.24$ \\
\hline$\%$ change & -0.35 & -0.35 & -0.13 & -0.12 & -0.0 & 0.07 & -0.1 & -0.2 \\
\hline Lowest-VOT Destination & $\$ 0.07$ & $\$ 0.02$ & $\$ 0.11$ & $\$ 0.08$ & $\$ 0.13$ & $\$ 0.11$ & $\$ 0.13$ & $\$ 0.12$ \\
\hline$\%$ change & -0.78 & -0.93 & -0.65 & -0.73 & -0.58 & -0.62 & -0.58 & -0.59 \\
\hline
\end{tabular}

Note: This table summarizes estimates of per-trip costs of three-minute delays by time of day. Each column aggregates results into three-hour bins so that, for example, 3:00am refers to the period between 12:01am-3:00am.

Table 8 summarizes the heterogeneity in travel costs after scaling by $\alpha$. Panel A displays the baseline cost as the mean value of time for a three minute delay with fifty percent weights on both VOT and $\delta \cdot$ VOT, or $\$ 0.30$ per trip. This value represents our best estimate of the average cost per-trip of any delay, equivalent to $\$ 6$ per hour, or around 60 percent of mean wages in Prague. Panel B shows the average costs, which we now allow to vary by time-of-day. Costs during the middle of the day are around 20 percent higher than in our uniform cost baseline, and costs in the evening are 20 percent lower. The second row of Panel B re-weights trip-costs by the overall share of Prague traffic volume by time-of-day, which we obtained from the Prague Transportation Yearbook. This row takes into account that traffic volume is very different during different hours, and thus if Prague were to apply time-of-day rules or incentives to construction, the hours from 9pm-6am would only cost 10-20\% of the short-run welfare lost to a similar delay in mid-day. Panel $\mathrm{C}$ further decomposes costs into different routes. It reports the mean cost of trips ending in the highest-, median-, and lowest-VOT destinations. The highest-VOT destination trips are 2-4 times as expensive as the lowest-VOT destination trips, which would lead the uniform baseline to over-estimate by nearly double (93\%) in 
some times-of-day and locations and under-estimate by up to 40 percent in other timesof-day and places. Panels B and C thus illustrate that there could be significant saving from timing road closures optimally, and from tailoring fines and rewards to projects based on their locations and the hours of closure.

Figure 10: Estimated Per-Trip Closure Costs by Route

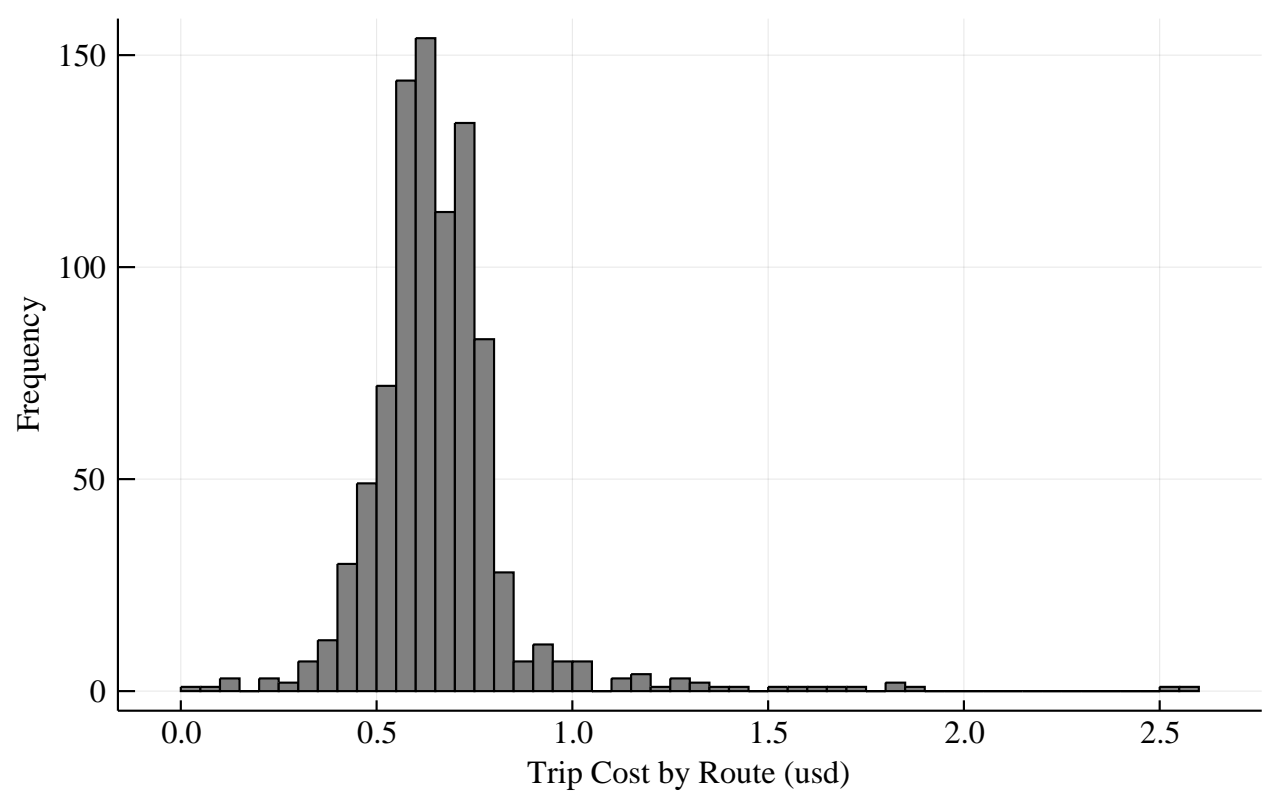

Note: This histogram shows route-specific estimates of mean per-trip costs of a three-minute delay across each of 900 distinct routes.

While Table 8 summarizes the importance of the underlying heterogeneity and of time in particular, costs are borne on a route-by-route basis. Since we estimate valuations over 30 origins and destinations, Figure 10 shows the mean per-trip costs of delays across each of 900 routes. In this figure we report weighted averages over time of day and person-specific variation. This is the appropriate metric for measuring delay costs when closures cannot be conducted during selected times of day. Even projects that require closures throughout the day, such as road re-paving, exhibit substantial heterogeneity in the social cost imposed on road users. To maintain the right incentives and avoid lengthy closures that would result in high delay costs, procurers might need to impose fines that vary according to the impacted routes.

Using information on the average daily traffic flows across a few key routes in the city, we can derive some illustrative examples. We pair reported traffic flows with our estimated delay cost in locations connected by each route and compute the weighted average vот over the associated trips. For example, a hypothetical three-minute delay along the Zlichovsky tunnel, which sees an average of 84,000 cars per day, would impose social costs from $\$ 31,600$ to $\$ 35,500$ per day depending on the composition of 
the direction of travel. A delay along the nearby Brusnicky tunnel, which sees 77,000 cars per day, would impose social costs from $\$ 29,600$ to $\$ 31,800$ per day. In contrast, by assuming a uniform average value of time of $\$ 0.30$ per car trip, the costs of these two hypothetical delays are $\$ 25,200$ and $\$ 23,100$ respectively. The differences could lead the city to under-price time-savings related to construction on these specific routes by up to $40 \%$, or about $\$ 300,000$ for a 30 -day span.

These results underscore the importance of using economically relevant VOT measurements when designing procurement incentives. The ability to draw insights from market behavior to quantify the costs of delays can be valuable for local policy makers and regulators. In order to determine what incentives to offer, our method could be used to measure the costs of delays due to each additional day of construction, as long as anticipated delay time estimates for the particular project are available. Our cost estimates can also be used in determining the value of new infrastructure, such as the benefits from adding a new lane to a road, upgrading an intersection, or adding public transportation services. In each of these cases we would need to have information about the specific project to explicitly characterize its impact on traffic speeds and any spillover effects to nearby roads, but an equilibrium analysis quantifying the costs and benefits would require the same type of time valuations that we have obtained with our approach.

\section{Conclusions}

The trade-off between time and money is at the heart of many important economic decisions. In this paper we use data from a large European ride share platform that offers menus with explicit trade-offs between time and money. We use this unique feature to estimate a demand model based on choices from these menus. This estimation allows us to recover riders' preferences and demand elasticities over time and money as well as their implied willingness-to-pay to reduce waiting time. We provide a framework to translate the estimates from this demand model into the implied value of time across different locations, times-of-day, and types of individuals.

Our demand model reveals noteworthy patterns in how individuals value time and money. Consumers are substantially more price elastic than waiting time elastic. There is large variation in the willingness to pay for waiting time reductions in the population of riders. We show how these differences vary throughout the day; for instance, we show that the willingness to pay is higher during work hours. We find that our NVOT measures are highly correlated with travel flows across routes, justifying the use of travel flows to measure the relative attractiveness of different places. 
We decompose the demand model estimates to quantify the value of time. We again find substantial heterogeneity by location, time-of-day, and individuals. A variance decomposition reveals that $79 \%$ of the overall heterogeneity is driven by individual differences. Once individual differences are accounted for, only a small fraction of the variation is due to location and time of day. In contrast with the literature on residential sorting, our results suggest that people with a higher value of time do not sort into locations with a higher value of time. This suggests that the valuation of places in the short-run may be more egalitarian than that implied by long-run decisions in housing markets.

By highlighting the underlying heterogeneity in the value of time, we show how our method can be used to target optimal procurement incentives in a more fine-grained way than standard industry practice. By combining our estimates with information from Prague's 2018 Transportation Yearbook, we show that using a uniform, city-wide measure of vот to infer the costs associated with road closures would mis-price delays by up to ninety percent along some routes.

Although our approach relies in part on unique features of our platform, we believe that it could be adapted to conduct a similar study within the US, for example with data from Uber or Lyft. Our approach shows that data generated by ride hail companies and other private transportation services can be exploited to better understand urban infrastructure and urban economic activity more broadly. 


\section{Bibliography}

Abowd, John M, Francis Kramarz, and David N Margolis, "High wage workers and high wage firms," Econometrica, 1999, 67 (2), 251-333.

Abrantes, Pedro AL and Mark R Wardman, "Meta-analysis of UK values of travel time: An update," Transportation Research Part A: Policy and Practice, 2011, 45 (1), 1-17.

Aguiar, Mark and Erik Hurst, "Life-cycle prices and production," American Economic Review, 2007, 97 (5), 1533-1559.

_ , _ , and Loukas Karabarbounis, "Recent developments in the economics of time use," Annu. Rev. Econ., 2012, 4 (1), 373-397.

Allen, Treb and Costas Arkolakis, "The welfare effects of transportation infrastructure improvements,” Technical Report, National Bureau of Economic Research 2019.

Almagro, Milena and Tomás Domınguez-Iino, "Location Sorting and Endogenous Amenities: Evidence from Amsterdam,” 2019.

Athey, Susan, Billy Ferguson, Matthew Gentzkow, and Tobias Schmidt, "Experienced Seggregation," 2019.

Becker, Gary S, "A Theory of the Allocation of Time," The Economic Journal, 1965, pp. 493-517.

_ , "A theory of marriage: Part I," Journal of Political economy, 1973, 81 (4), 813-846.

Belenky, Peter, "Revised departmental guidance on valuation of travel time in economic analysis," US Department of Transportation. Washington, DC, 2011.

Bento, Antonio, Kevin Roth, and Andrew R Waxman, "Avoiding traffic congestion externalities? the value of urgency," Technical Report, National Bureau of Economic Research 2020.

Bloom, Nicholas, James Liang, John Roberts, and Zhichun Jenny Ying, "Does working from home work? Evidence from a Chinese experiment," The Quarterly Journal of Economics, 2015, 130 (1), 165-218.

Bonhomme, Stéphane, Thibaut Lamadon, and Elena Manresa, "Discretizing unobserved heterogeneity," University of Chicago, Becker Friedman Institute for Economics Working Paper, 2017, (2019-16). 
Borjesson, Maria, Mogens Fosgerau, and Staffan Algers, "On the income elasticity of the value of travel time," Transportation Research Part A: Policy and Practice, 2012, 46 (2), $368-377$.

Brancaccio, Giulia, Myrto Kalouptsidi, and Theodore Papageorgiou, "Geography, Search Frictions and Endogenous Trade Costs," 2017.

_, , , , and Nicola Rosaia, "Search Frictions and Efficiency in Decentralized Transport Markets,” Technical Report, mimeo, Harvard University 2020.

Buchholz, Nicholas, "Spatial Equilibrium, Search Frictions and Dynamic Efficiency in the Taxi Industry," 2018.

_ , Laura Doval, Jakub Kastl, Filip Matejka, and Tobias Salz, "Supplement to "The Value of Time: Evidence From Auctioned Cab Rides”," 2020. Click here.

Camerer, Colin, Linda Babcock, George Loewenstein, and Richard Thaler, "Labor supply of New York City cabdrivers: One day at a time," The Quarterly Journal of Economics, 1997, pp. 407-441.

Castillo, Juan Camilo, "Who Benefits from Surge Pricing?," Available at SSRN 3245533, 2019.

Chen, M Keith, Judith A Chevalier, Peter E Rossi, and Emily Oehlsen, "The value of flexible work: Evidence from uber drivers," Technical Report, National Bureau of Economic Research 2017.

Chetty, Raj, John N Friedman, Nathaniel Hendren, Maggie R Jones, and Sonya R Porter, "The opportunity atlas: Mapping the childhood roots of social mobility," Technical Report, National Bureau of Economic Research 2018.

Cirillo, Cinzia and Kay W Axhausen, "Evidence on the distribution of values of travel time savings from a six-week diary," Transportation Research Part A: policy and practice, 2006, 40 (5), 444-457.

Cohen, Peter, Robert Hahn, Jonathan Hall, Steven Levitt, and Robert Metcalfe, "Using big data to estimate consumer surplus: The case of uber," Technical Report, National Bureau of Economic Research 2016.

Couture, Victor, Cecile Gaubert, Jessie Handbury, and Erik Hurst, "Income growth and the distributional effects of urban spatial sorting," Technical Report, National Bureau of Economic Research 2019. 
_, Gilles Duranton, and Matthew A Turner, "Speed," Review of Economics and Statistics, 2018, 100 (4), 725-739.

Crawford, Vincent $\mathbf{P}$ and Juanjuan Meng, "New york city cab drivers' labor supply revisited: Reference-dependent preferences with rationalexpectations targets for hours and income," The American Economic Review, 2011, 101 (5), 1912-1932.

Davis, Donald R, Jonathan I Dingel, Joan Monras, and Eduardo Morales, "How Segregated is Urban Consumption?," 2017.

Domencich, Thomas A and Daniel McFadden, "Urban travel demand-a behavioral analysis," Technical Report 1975.

Dunning, Iain, Joey Huchette, and Miles Lubin, "JuMP: A modeling language for mathematical optimization," SIAM Review, 2017, 59 (2), 295-320.

Duranton, Gilles and Matthew A Turner, "The fundamental law of road congestion: Evidence from US cities," American Economic Review, 2011, 101 (6), 2616-52.

Eeckhout, Jan and Philipp Kircher, "Assortative matching with large firms," Econometrica, 2018, 86 (1), 85-132.

Engelson, Leonid and Mogens Fosgerau, "The cost of travel time variability: three measures with properties," Transportation Research Part B: Methodological, 2016, $91,555-564$.

Fajgelbaum, Pablo D and Edouard Schaal, "Optimal transport networks in spatial equilibrium,” Technical Report, National Bureau of Economic Research 2017.

Farber, Henry S., "Is Tomorrow Another Day? The Labor Supply of New York City Cabdrivers," The Journal of Political Economy, 2005, 113 (1), 46-82.

Farber, Henry S, "Reference-dependent preferences and labor supply: The case of New York City taxi drivers," The American Economic Review, 2008, 98 (3), 10691082.

Fosgerau, Mogens, Katrine Hjorth, and Stéphanie Vincent Lyk-Jensen, “The Danish Value of Time Study," Technical Report, Danmarks Transportforskning, Report $5,2007$.

Frechette, Guillaume R, Alessandro Lizzeri, and Tobias Salz, "Frictions in a competitive, regulated market: Evidence from taxis," Technical Report, National Bureau of Economic Research 2018. 
Hall, Jonathan D, "Pareto improvements from Lexus Lanes: The effects of pricing a portion of the lanes on congested highways," Journal of Public Economics, 2018, $158,113-125$.

Hall, Jonathan V, John J Horton, and Daniel T Knoepfle, "Pricing Efficiently in Designed Markets: The Case of Ride-Sharing,” 2019.

Heblich, Stephan, Stephen J Redding, and Daniel M Sturm, "The making of the modern metropolis: evidence from London," Technical Report, National Bureau of Economic Research 2018.

Jara-Diaz, Sergio R., Marcela A. Munizaga, Paulina Greeven, Reinaldo Guerra, and Kay Axhausen, "Estimating the value of leisure from a time allocation model," Transportation Research Part B: Methodological, 2008, 42 (10), 946 - 957.

Kreindler, Gabriel E, “The welfare effect of road congestion pricing: Experimental evidence and equilibrium implications," Unpublished paper, 2018.

Kreindler, GE and Y Miyauchi, "Measuring commuting and economic activity inside cities with cell phone records," Technical Report, Working paper 2019.

Lam, Terence C. and Kenneth A. Small, "The value of time and reliability: measurement from a value pricing experiment," Transportation Research Part E: Logistics and Transportation Review, 2001, 37 (2), 231 - 251. Advances in the Valuation of Travel Time Savings.

Lewis, Gregory and Patrick Bajari, "Procurement contracting with time incentives: Theory and evidence," The Quarterly Journal of Economics, 2011, 126 (3), 11731211.

Liu, Tracy, Zhixi Wan, and Chenyu Yang, "The efficiency of a dynamic decentralized two-sided matching market," Available at SSRN 3339394, 2019.

Mas, Alexandre and Amanda Pallais, "Valuing alternative work arrangements," American Economic Review, 2017, 107 (12), 3722-59.

McFadden, Daniel, "The measurement of urban travel demand," Journal of public economics, 1974, 3 (4), 303-328.

Miller, Ted R, The Value of Time and the Benefit of Time Saving: A Literature Synthesis and Recommendations on Values, Urban Institute, 1989. 
Nevo, Aviv and Arlene Wong, "The elasticity of substitution between time and market goods: Evidence from the Great Recession," International Economic Review, 2019, $60(1), 25-51$.

Petrin, Amil and Kenneth Train, "A control function approach to endogeneity in consumer choice models," Journal of marketing research, 2010, 47 (1), 3-13.

Research, John Bates Services Significance Quantitative, "Values of time and reliability in passenger and freight transport in The Netherlands," Technical Report 2007.

Rossi, Peter E. and Greg M. Allenby, "Bayesian Statistics and Marketing," Marketing Science, 2003, 22, pp. 304-329.

_ ,_, and Rob McCulloch, Bayesian Statistics and Marketing, Wiley, 2005.

Shimer, Robert and Lones Smith, "Assortative matching and search," Econometrica, 2000, 68 (2), 343-369.

Small, Kenneth A, "The scheduling of consumer activities: work trips," The American Economic Review, 1982, 72 (3), 467-479.

Small, Kenneth A., "Valuation of travel time," Economics of Transportation, 2012, 1 (1), $2-14$.

Su, Yichen, “The Rising Value of Time and the Origin of Urban Gentrification,” Technical Report, Stanford University 2018.

Tanner, Martin A. and Wing Hung Wong, "The Calculation of Posterior Distributions by Data Augmentation," Journal of the American Statistical Association, 1987, 82 (398), 528-540.

Thakral, Neil and Linh T Tô, "Daily Labor Supply and Adaptive Reference Points," 2017.

Train, Kenneth E, Discrete Choice Methods with Simulation, Cambridge University Press, 2009.

Tseng, Yin-Yen and Erik T Verhoef, "Value of time by time of day: A statedpreference study," Transportation Research Part B: Methodological, 2008, 42 (7-8), 607-618.

Vickrey, William S, "Congestion theory and transport investment," The American Economic Review, 1969, 59 (2), 251-260. 
Waldfogel, Joel, "The selection hypothesis and the relationship between trial and plaintiff victory," Journal of Political Economy, 1995, 103 (2), 229-260. 


\section{Online Appendix}

\section{A Omitted Graphics}

Figure 11: Picture of the Liftago App

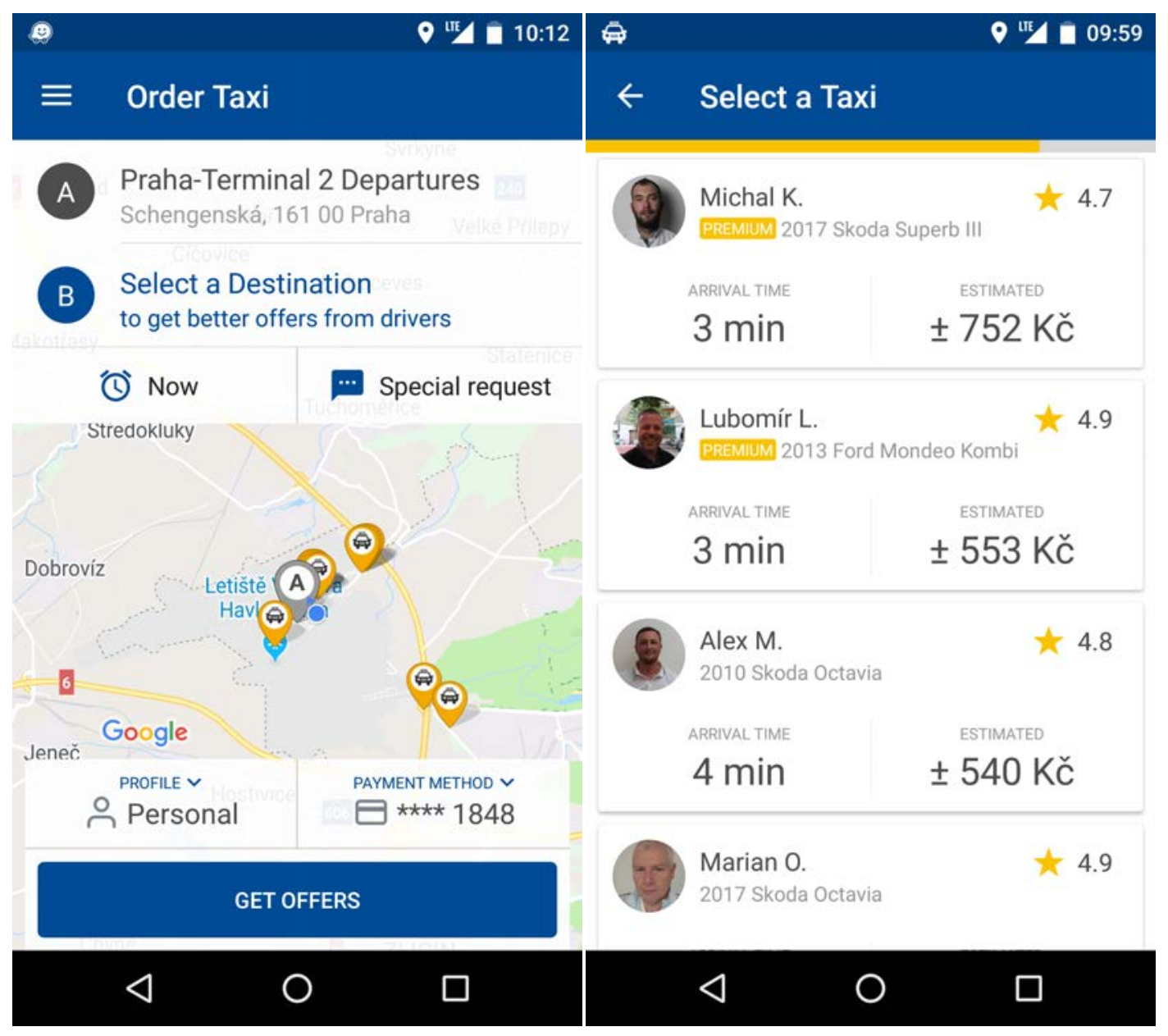

NotE: This Graph shows the interfact of the Liftago App. 
Figure 12: Trips with Price/Waiting Tradeoff

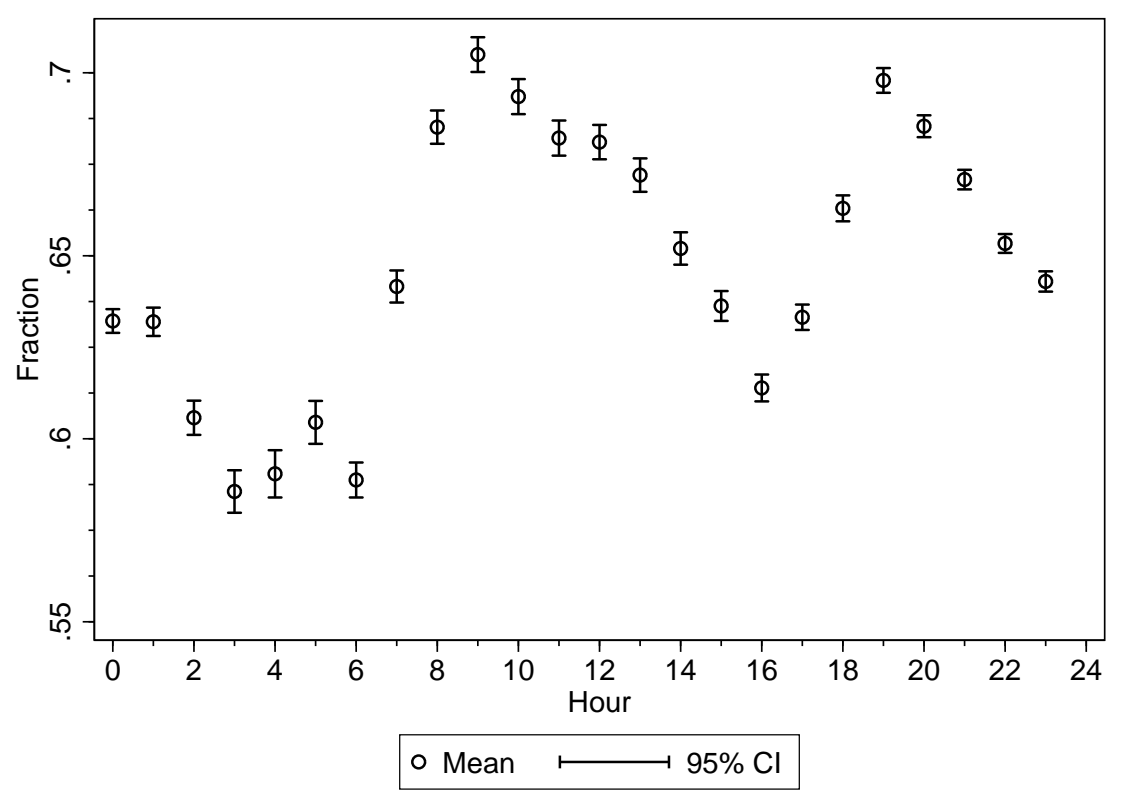

Note: The graph shows the proportion of trips that involve an option to spend more and wait less.

Figure 13: Driver Fixed Effects

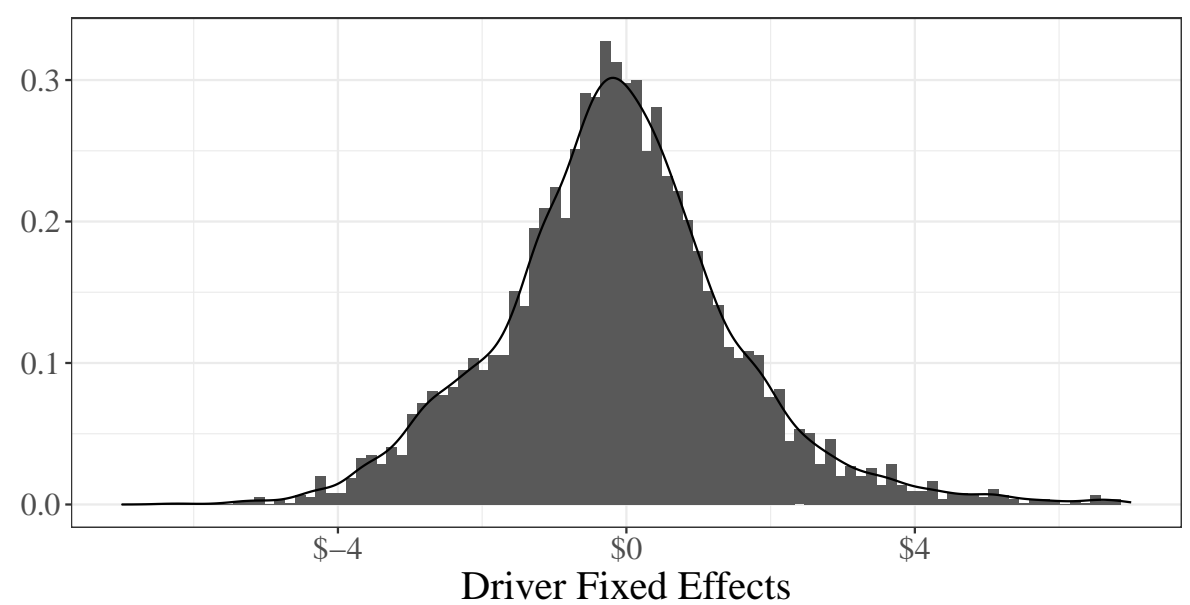


Figure 14: Choices as a Function of Wait Time

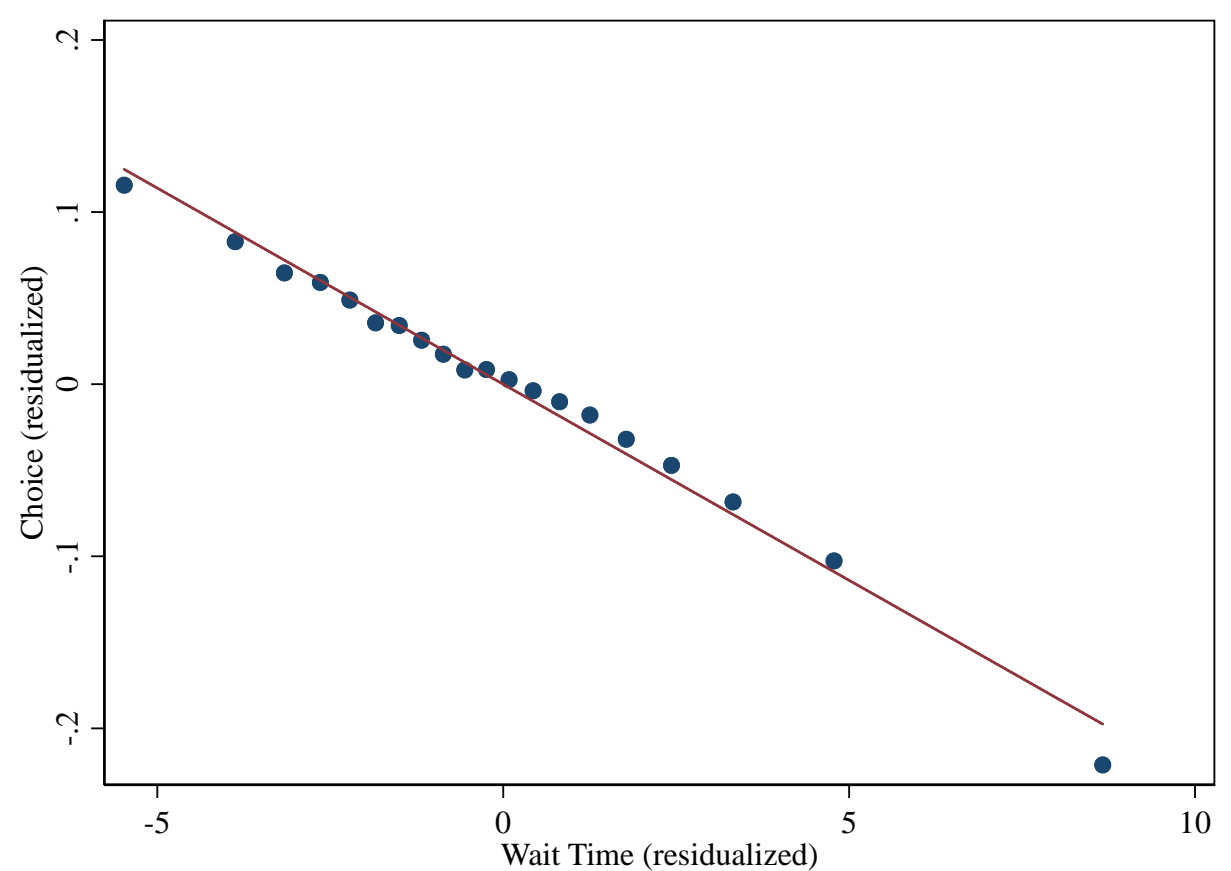

(a) Trips Choice as Function of Waiting Time

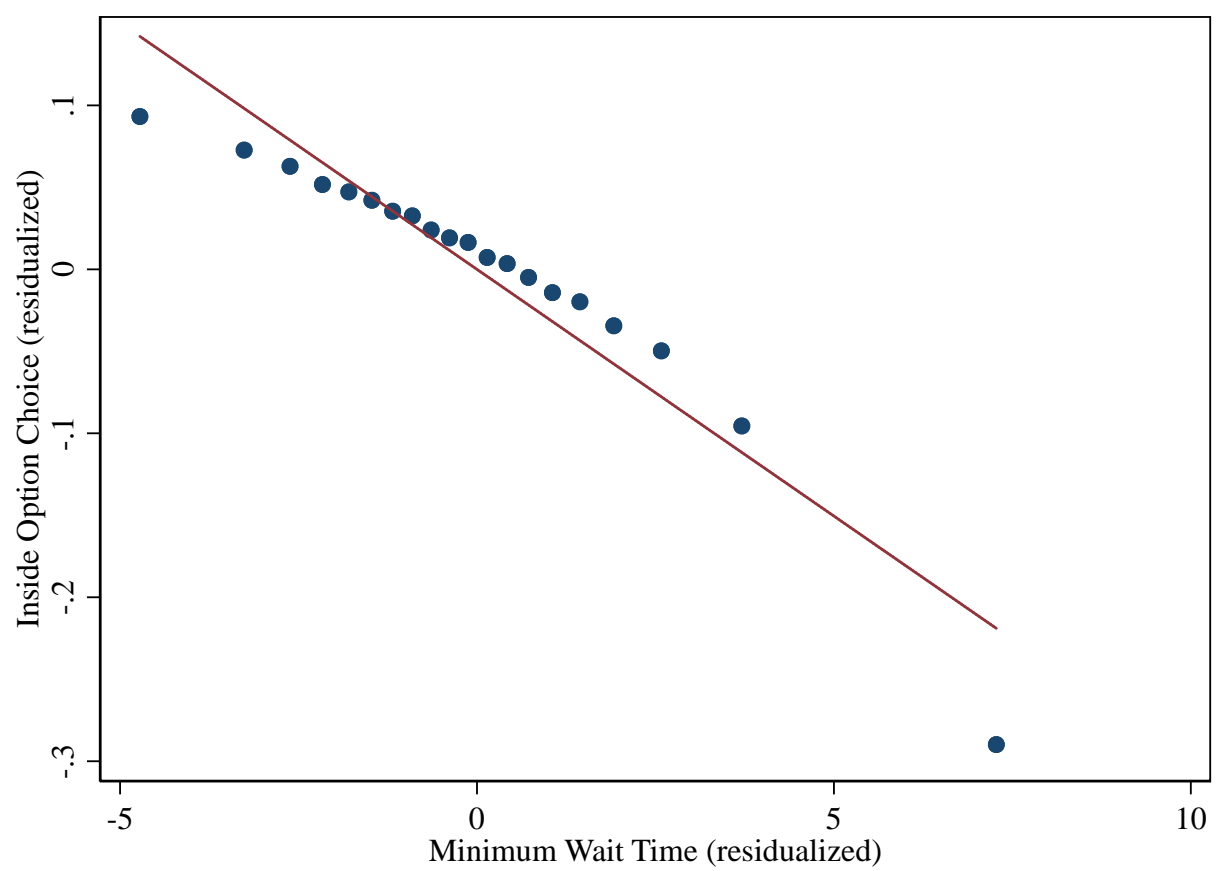

(b) Inside Option Choice as Function of the Minimum Waiting Time

NotE: The graph on top shows the probability of choosing a trip as a function of the wait time, both residualized after taking into accound time of day, origin and destination location, weather conditions, and the respective other prices and wait times. The graph on the bottom shoes the probability of choosing any trip over the outside option as a function of the minimum waiting time, both residualized for time of day, origin and destination locations, and weather conditions. 


\section{B Relation to the model in Small (1982)}

The model described in Equation 1 is the workhorse model of the transportation literature on travel time savings (see, e.g., Tseng and Verhoef (2008) and Engelson and Fosgerau (2016)). It has its origins in the work of Small (1982), which is inspired by the work of of Vickrey (1969). Small (1982) considers a special case of the above model where: ${ }^{27}$

$$
\begin{aligned}
\operatorname{VOT}^{o}(t) & =\alpha \\
\operatorname{VOT}^{d} & =\alpha-\beta \mathbb{1}\left[t \leq t^{*}\right]+\gamma \mathbb{1}\left[t \geq t^{*}\right]
\end{aligned}
$$

Note that under this specification, $\alpha=\operatorname{voT}^{o}, \beta=\left(\operatorname{voT}^{o}(t)-\operatorname{voT}^{d}(t)\right) \mathbb{1}\left[t \leq t^{*}\right]$, and $\gamma=$ $\left(\operatorname{voT}^{d}(t)-\operatorname{voT}^{o}(t)\right) \mathbb{1}\left[t>t^{*}\right]$. s This version of the model, where over the relevant time

Figure 15: Value at origin (black) and destination (blue) as a function of time in the constant model

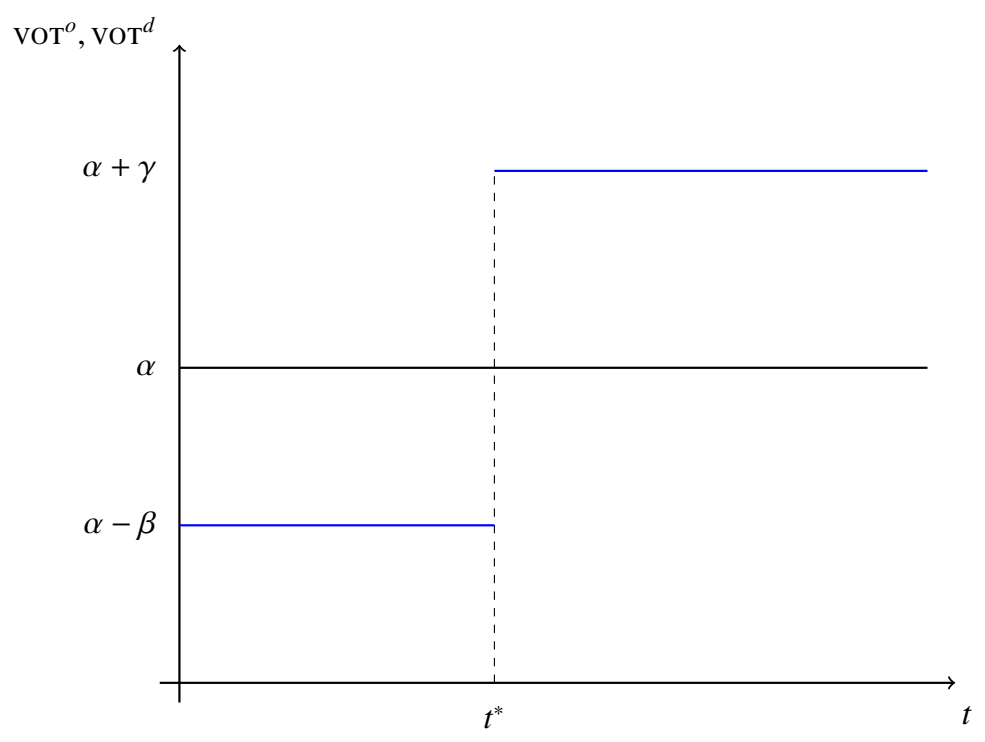

intervals, $\operatorname{VOT}^{o}$ and $\operatorname{voT}^{d}$ are constant, is the one to which the approximation described in Section 3 maps to. Whereas Small (1982), assumes that voT ${ }^{o}$ and voT ${ }^{d}$ are constant everywhere, it is important to note that this is not the case in the exercise that we carry out. Indeed, we only assume that this holds over $[q, q+w+\Delta]$; however, we allow these values to vary across different times of day, different locations, and different types of riders.

${ }^{27}$ Small (1982) is expressed in terms of opportunities cost of time. Equation 15 in terms of the values is equivalent (see Tseng and Verhoef (2008) and Engelson and Fosgerau (2016)). 


\section{B.1 Optimal query time}

In reality, riders may plan the time at which they request a ride. Thus, the rider's problem is given by:

$$
\max _{q} \mathbb{E}\left[\max _{i \in \mathcal{I}} u\left(q, w_{i}\right)-p_{i}\right]
$$

where the expectation is over the price and waiting times that the rider will face. Let $u^{*}\left(q,\left(w_{i}, p_{i}\right)_{i \in \mathcal{I}}\right)$ denote the term inside the expectation and let $q^{*}$ denote the solution to the problem in Equation 16. Note that what we observe in the data corresponds to $u^{*}\left(q^{*},\left(w_{i}, p_{i}\right)_{i \in I}\right)$. In what follows, we show that in spite of this there are two versions of the model where the relevant parameters are identified, and hence the values of time, for all $q$ and not just $q^{*}$. Furthermore, to convey the main ideas we focus on the case in which $w_{2}, p_{2}, p_{1}$ are known, and the rider just faces uncertainty about the ETA of the first ride, $w_{1} \sim F$.

The analysis that follows implicitly assumes that conditional on the trip, all riders are identical. Buchholz et al. (2020) shows how to extend the arguments presented here to the case in which, conditional on a trip, there is heterogeneity in the preferences of the riders who need to take that trip.

\section{B.1.1 Small (1982)}

Consider first the specification in Small (1982), described in Equation 15. We assume that $t^{*} \leq T$. Fix a query time $q \geq 0$ such that $q+\Delta<t^{* 28}$. Figure 16 plots the function $u\left(q, w_{2}\right)+p_{\Delta}::^{29}$

In a world without uncertainty in which the rider anticipates the waiting time $w$, she would simply choose $q=t^{*}-(\Delta+w)$, so that the effective function (given the optimal choice of $q^{*}$ ) would be always decreasing. ${ }^{30}$

Given a waiting time $w_{2}$, we can think of the waiting times $w_{1}$ that are chosen over $w_{2}$ for a given price differential $p_{\Delta}$,

$$
C\left(w_{2}, p_{\Delta}\right)=\left\{w_{1}: u\left(q, w_{1}\right) \geq u\left(q, w_{2}\right)+p_{\Delta}\right\}
$$

\footnotetext{
${ }^{28}$ We show below that the optimal query time always satisfies this, so this is the relevant case

${ }^{29}$ The figure implicitly assumes that the price differential is not "very" negative so that

$$
p_{\Delta}+\alpha(T-\Delta)+\gamma\left(T-t^{*}\right) \geq 0 .
$$

Otherwise, the entire figure would be below the $w$-axis.

${ }^{30}$ To see this, note that even though the above is the picture as a function of $w$, for fixed $w$, we would have the same picture as a function of $q$, once we flip the roles of $q$ and $w$.
} 
Figure 16: The function $u\left(q, w_{2}\right)+p_{\Delta}$

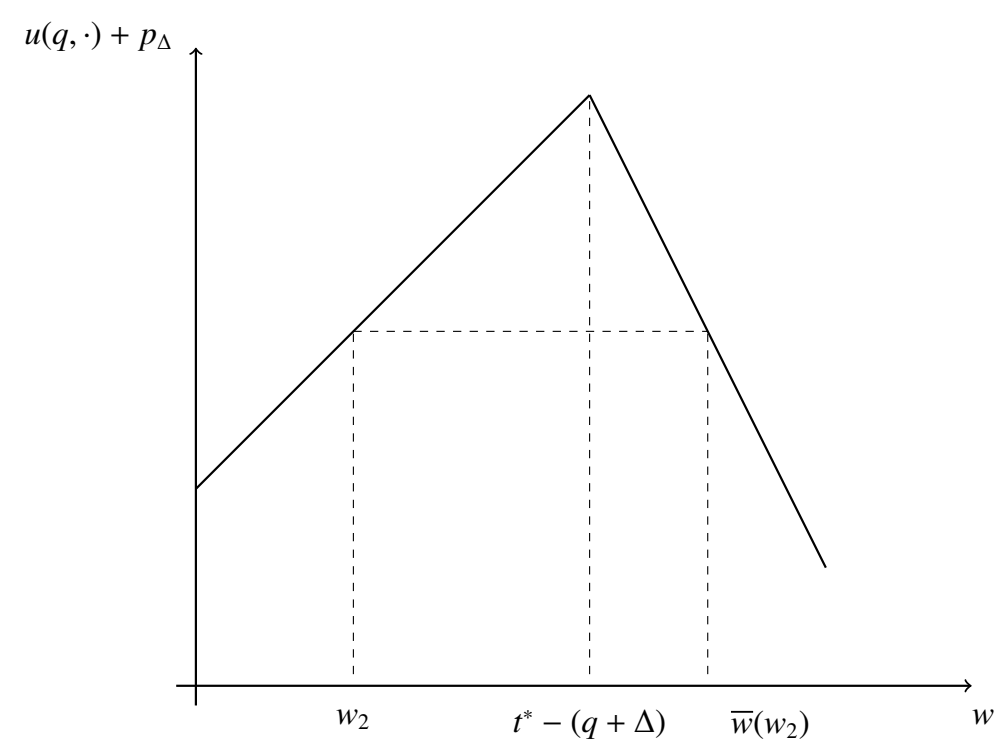

As Figure 16 suggests, $C\left(w_{2}, p_{\Delta}\right)$ is an interval with one of its endpoints being $w_{2}$. Intuitively, if the consumer arrives before their ideal time under $w_{2}$, then $w_{2}$ is the lowest ETA that the consumer is willing to accept instead of trip 2 given $p_{\Delta}$. Similarly, if the consumer arrives after their ideal time under $w_{2}$, then $w_{2}$ is the highest ETA that the consumer is willing to take instead of trip 2 given $p_{\Delta}$. Thus, the shape of $C\left(w_{2}, p_{\Delta}\right)$ reveals whether $w_{2}$ is below or above $t^{*}-(q+\Delta){ }^{31}$

To infer the parameters $\beta, \gamma$, consider the case in which $p_{\Delta}=0$. Fix $w_{2}$ such that $\min \left\{w: w \in C\left(w_{2}, 0\right)\right\}=w_{2}$. For $\epsilon>0, \beta$ is identified as the parameter such that the rider is indifferent between $\left(w_{2}, p\right)$ and $\left(w_{2}-\epsilon, p-\beta \epsilon\right)$. That is, $\beta \epsilon$ is how much we have to pay the rider to take a trip that leaves $\epsilon>0$ earlier than trip 2. On the other hand, $\gamma$ is identified as the parameter such that the rider is indifferent between $\left(w_{2}, p\right)$ and $\left(w_{1}+\epsilon, p-\gamma \epsilon\right)$. That is, $\gamma \epsilon$ is how much we have to pay the rider to take a trip that leaves $\epsilon>0$ later than trip 2 .

${ }^{31}$ Formally, one can show that if $w_{2}<t^{*}-(q+\Delta)$, then $C\left(w_{2}, p_{\Delta}\right)=\left[w_{2}, \bar{w}_{2}\left(w_{2}, q, p_{\Delta}\right)\right]$ where

$$
\bar{w}\left(w_{2}, q, p_{\Delta}\right)=\max \left\{\frac{p_{\Delta}+(\beta+\gamma)\left(t^{*}-(q+\Delta)\right)-\beta w_{2}}{\gamma}, t^{*}-(q+\Delta)\right\},
$$

whereas if $w_{2}$ is to the right of $t^{*}-(q+\Delta)$, then $C\left(w_{2}, p_{\Delta}\right)=\left[\underline{w}_{2}\left(w_{2}, q, p_{\Delta}\right), w_{2}\right]$, where

$$
\underline{w}\left(w_{2}, q, p_{\Delta}\right)=\min \left\{\frac{p_{\Delta}+(\beta+\gamma)\left(t^{*}-(q+\Delta)\right)-\gamma w_{2}}{\beta}, t^{*}-(q+\Delta)\right\} .
$$


Finally, note that with $\beta, \gamma$ identified, we can identify $t^{*}-(q+\Delta)$ from solving:

$$
\frac{\beta w_{2}+\gamma \bar{w}\left(w_{2}, q, 0\right)}{\beta+\gamma}
$$

where $\bar{w}\left(w_{2}, q, 0\right)$ is the maximum element of $C\left(w_{2}, p_{\Delta}\right)$. A similar experiment identifies $\beta, \gamma$ and $t^{*}-q$ when $w_{2}=\max \left\{w: w \in C\left(w_{2}, p_{\Delta}\right)\right\}$. Clearly, $\alpha$ cannot be identified.

When $q+\Delta \geq t^{*}$, then the rider is always late. This would correspond to a case where Figure 16 only displays the downward sloping branch. In this case, the rider always prefers shorter ETAs for a given price differential. However, one can show that in this model the rider always chooses $q^{*}$ so that $q^{*}+\Delta<t^{*}$ :

Proposition 1. Let $\operatorname{VOT}^{o}$, $\operatorname{voT}^{d}$ be as in Equation 15. Then, the optimal choice of query time, $q^{*}$, satisfies that $q^{*}+\Delta<t^{*}$.

The proof is available upon request. However, it is intuitive that such a result should be true. Inspection of Figure 16 reveals that the rider is risk averse in $w$; by choosing $q$ smaller than $t^{*}-\Delta$, the rider makes sure that he has a chance to arrive early.

\section{B.1.2 Linear specification}

Another common specification of the model is the linear case (see Engelson and Fosgerau (2016)):

$$
\begin{aligned}
\operatorname{vOT}^{o}(t) & =\alpha, \\
\operatorname{vOT}^{d}(t) & =\alpha+\gamma\left(t-t^{*}\right),
\end{aligned}
$$

where $\alpha, \gamma>0$ and $t^{*}$ is the desired arrival time. We continue to assume that the trip length, $\Delta$ is less than $t^{*}$ so that the rider can potentially arrive at her ideal arriving time.

As before, it will not be possible to identify $\alpha$ from the choices. However, a similar exercise allows us to identify $\gamma, t^{*}$. Figure 17 plots the function $u(\cdot, q)+p_{\Delta}$ for $q<t^{*}-\Delta$ :

To understand how choices reveal $\gamma, t^{*}$, consider the case in which $p_{\Delta}=0$. When $2 t^{*}<T+\Delta+q$, we have that $C\left(w_{2}, p_{\Delta}\right)$ is an interval with $w_{2}$ as its lower or upper end (see Figure 17a). Moreover, $T-t^{*}$ is always the center of that interval. Thus, we can identify $T-t^{*}$ from the midpoint of this interval. Given $T-t^{*}$, we can write

$$
u\left(q, w_{2}\right)=\frac{\gamma}{2}\left(w_{2}-\left(T-t^{*}\right)\right)^{2}+u\left(T-t^{*}, q\right)
$$


Figure 17: The function $u\left(q, w_{2}\right)+p_{\Delta}$

(a) $2 t^{*}<T+\Delta+q$

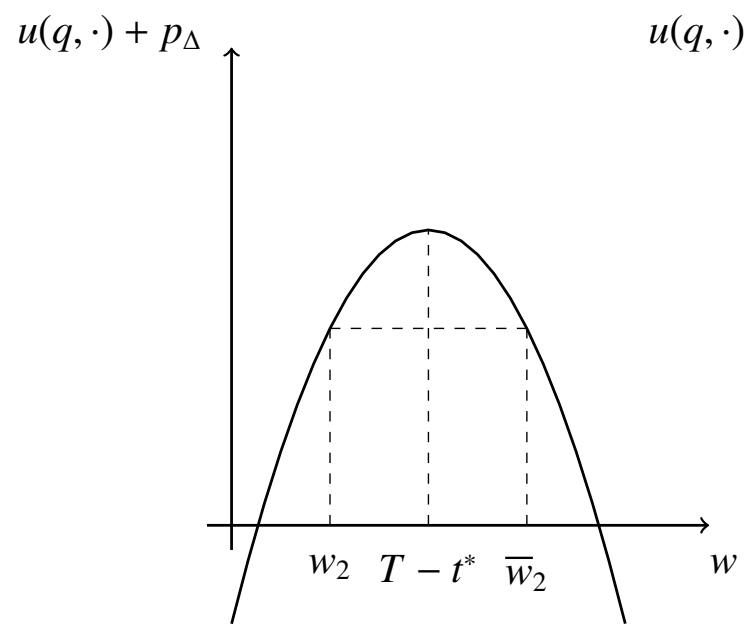

(b) $2 t^{*}>T+q+\Delta$

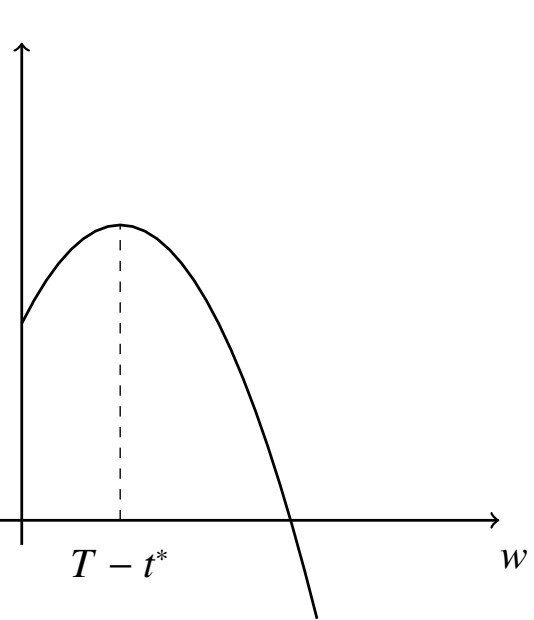

Using this, let $k$ be such that the rider is indifferent between $\left(w_{2}, p\right)$ and $\left(w_{2}-\epsilon, p-k \epsilon\right)$. Then, it follows that

$$
\frac{\gamma}{2}=\frac{k}{2\left(w_{2}-\left(T-t^{*}\right)\right)-\epsilon}
$$

On the other hand, when $2 t^{*}>T+q+\Delta$, the function $u(\cdot, q)+p_{\Delta}$ is as in Figure $17 \mathrm{~b}$. For $p_{\Delta}=0$ and $w_{2}$ low enough, however, $C\left(w_{2}, 0\right)$ is an interval with $w_{2}$ as its lower bound and $T-t^{*}$ as its midpoint. Thus, a similar experiment identifies $\gamma / 2$.

The above discussion implicitly assumes that $q<t^{*}-\Delta$. Indeed, this is without loss of generality:

Proposition 2. Let $\operatorname{VOT}^{o}$, $\operatorname{voT}^{d}$ be as in Equation 18. Then, the optimal choice of query time, $q^{*}$, satisfies that $q^{*}+\Delta<t^{*}$.

The proof is available upon request.

\section{Identification of Location Values}

The following are the three assumptions that will be used in our main theorem below.

Assumption 1. (Independence across locations) $F\left(V_{a}^{i, k}, V_{a^{\prime}}^{i, k^{\prime}}\right)=F_{i, a, k}(V) \cdot F_{i, a^{\prime}, k^{\prime}}(V)$ $\forall\left(a, a^{\prime}\right) \in \mathcal{J}^{2}$ and $\left(k, k^{\prime}\right) \in\{o, d\}^{2}$

The following assumption imposes the existence of at least one location where the mean of the values is known either when this location is the destination or it is the 
origin. For example, the mean (local) wage per minute might be a good approximation of the mean value per minute for trips originating in a typical business area.

Assumption 2. (Location Normalization) $\exists a \in \mathcal{A}, k \in\{o, d\}: \mathbb{E}\left[v_{i, a}^{k}\right]=\mu_{0}$.

While we will establish non-parametric identification of the value distributions, for the empirical application it will be useful to consider a special case of normal distributions.

Assumption 3. (Normal Distributions) $V_{i, a}^{k} \sim N\left(\mu_{a}^{k}, \sigma_{a}^{k}\right) \forall i, a \in \mathcal{A}, k \in\{o, d\}$ ), iid $\operatorname{across}(i, a, k)$.

With these assumptions we can state the following identification result. The first part is straightforward, but requires the knowledge of a subset of trips, such that one of the two values in the difference $V_{i, a}^{d}-V_{i, a^{\prime}}^{o}$ is a known constant. The second part imposes a potentially weaker requirement: only that we know the mean value at one location (whether it is the origin or the destination) and that one part of difference can be held constant across a subset of observations. The last part is a special case, which imposes more parametric structure.

Theorem 3. (Sufficient Conditions for Identification)

1. Under Assumption 1, if $\exists\left(X^{o}, X^{d}, a\right) \subset X \times X \times \mathcal{A}: \operatorname{Pr}\left[V_{i, a}^{k}=\hat{v}_{k} \mid X^{k}, a\right]=1$ for $k \in\{o, d\}$, then $F_{a, k}(v)$ is identified $\forall a \in \mathcal{A}, k \in\{o, d\}$.

2. Under Assumptions 1 and 2 , when either $\exists k \in\{o, d\}: v_{i, a, t}^{k}=v_{i^{\prime}, a, t}^{k} \forall\left(i, i^{\prime}, a, t\right)$ or $\exists k \in\{o, d\}: v_{i, a, t}^{k}=v_{i, a, t^{\prime}}^{k} \forall\left(i, a, t, t^{\prime}\right)$, then $F_{a, k}(v)$ is identified $\forall a \in \mathcal{A}$ and $k \in\{o, d\}$ whenever $|\mathcal{A}| \geq 3$.

3. Under Assumptions 2 and $3,\left(\mu_{a}^{k}, \sigma_{a}^{k}\right)$ is identified $\forall a \in \mathcal{A}, k \in\{o, d\}$ whenever $|\mathcal{A}| \geq 3$.

Proof: See Appendix C. The theorem says that we can identify the distributions of valuations non-parametrically whenever (1) values are independent across locations and we can isolate cases for which the value either at the origin or at the destination is known to be equal to some constant, (2) when values are independent and are locationand time-, but not individual-specific either at the origin or at the destination (e.g., everyone has the same value of spending a minute in a residential area) or values are location and individual, but not time-specific (e.g., an individual has always the same value of a minute of being at his business location at 8 am every Monday), we know the mean value in at least one location and there are at least 3 locations. Given the 
previous result, we can of course also identify the values parametrically. However, this approach is particularly easy when the values are iid normal, when there are at least 3 locations and we have one location normalization for both mean and variance. (1) follows from restricting attention to cases where one part of the difference is known to be a constant, and hence the distribution can be fully recovered from the distribution of the differences. (2) follows from a deconvolution argument as in Li and Vuong (1998) since under the hypothesis of the theorem we can construct a sample in which we hold one part of the difference fixed. By applying the Kotlarski Lemma, one can then recover the distributions of both pieces of the difference (up to the location). The additional location normalization pins down the means separately. (3) follows since adding an additional location to the existing set of $L$ locations comes with 4 new parameters (two means and two standard deviations) and generates $2 \cdot(L-1)$ additional equations and hence one needs $L \geq 3$ together with the two normalizations.

Proof. (1) Restricting attention to $X^{o}$ and trips originating in $a$ going to destination $a^{\prime}$ identifies $F_{D, a^{\prime}}(v)$ trivially from the observed distribution of the differences by shifting the distribution of the data by the relevant constant, $\hat{v}_{O}$. Similarly, $F_{O, a^{\prime}}(v)$ is identified from using only data from $X^{d}$ and trips that originated in $a$ and went to $a^{\prime}$.

(2) Consider $|\mathcal{A}|=3$ and the case $v_{i, a, t}^{o}=v_{i^{\prime}, a, t}^{o} \forall\left(i, i^{\prime}, a, t\right)$,i.e., that values at a given origin and time are the same for all individuals $i$. Since values at origin are the same, the deconvolution argument identifies (using variation over time) the distribution of values at origin and the distribution of the values at each destination (using variation across individuals going to each destination) - up to a location normalization, i.e., the two means cannot be identified separately directly. ${ }^{32}$ With 3 locations we can setup the following system of 6 equations in 6 unknowns (in the means of values at origin and at destination), where the objects on the LHS are recovered from the deconvolution argument: $\mu_{12}=-\mu_{1}^{o}+\mu_{2}^{d}, \mu_{13}=-\mu_{1}^{o}+\mu_{3}^{d}, \mu_{21}=-\mu_{2}^{o}+\mu_{1}^{d}, \mu_{23}=-\mu_{2}^{o}+\mu_{3}^{d}, \mu_{31}=$ $-\mu_{3}^{o}+\mu_{1}^{d}, \mu_{32}=-\mu_{3}^{o}+\mu_{2}^{d}$. This system is not identified since the matrix of coefficients has a rank of 5 . Substituting equation from Assumption 2 for one of the equations including that mean restores the full rank of the coefficient matrix. For any additional location, 2 new parameters and $L-1$ new equations are introduced, but the rank is still (2L-1). Substituting equation from Assumption 2 brings the rank to $2 \mathrm{~L}$, and we have $L \cdot(L-1)$ equations. Hence we can simply keep any subset with rank $2 \mathrm{~L}$ as at true parameters all of these equations will hold simultaneously.

\footnotetext{
${ }^{32}$ In the measurement literature setup $Y_{1}=X+\varepsilon_{1}, Y_{2}=X+\varepsilon_{2}$, the distributions $F(X), G(\varepsilon)$ are identified from $H\left(Y_{1}, Y_{2}\right)$ whenever the usual iid assumption holds, when the characteristic functions of $F$ and $\mathrm{G}$ are non-vanishing everywhere and when $E(\varepsilon)=0$.
} 
The case $v_{i, a, t}^{o}=v_{i, a, t^{\prime}}^{o} \forall\left(i, a, t, t^{\prime}\right)$, is analogous, except variation over individuals is used for the deconvolution argument and the distribution of values at origin and destination is identified using variation within individuals as they go to different destinations. The means are then recovered as above.

(3) Since sum of two normally distributed random variables is also normally distributed with mean being the sum of means and the variance being the sum of the variances, the means can be recovered directly by using the argument in (2). However, the system of equations involving the variances is also less than full rank - and hence to identify these directly, one needs another location normalization (such as the knowledge of the variance at some location) or one needs to appeal to case (2), which establishes that the variances are identified non-parametrically.

\section{Data Details}

\section{D.1 Locations}

Using the exact GPS points of trip origin, we partition our data into 30 locations. Figure 18 shows these locations together with an index value for comparing results in Section 5. The partitioning is done according to a simple $k$-means clustering procedure on the requested pickup locations with $k=30$. This procedure minimizes the straight-line distance between each point and the weighed center of all points within the same cluster, with the constraint that each cluster has an equal number of points. The depicted locations are close approximations, displayed as Voronoi cells that contain the clustered points. This process allows location definitions to be independent of any political boundaries and better representative of places in which demand is concentrated.

\section{D.2 Market Time Series Summary}

Figure 19, panels (a) and (b) summarize the week-over-week trends in prices. Beginning 2017 the ridership and drivers stop to grow and enter a relatively stationary period, although there are some large swings in the number of passengers towards the end of 2017. It shows the relationship between labor supply and price during the sample period. As expected, price decreases as supply increases during the winter holiday seasons and increases as supply falls during the summer. The second panel shows how average waiting time and average price evolve during the sample period. 
Figure 18: Locations in Prague

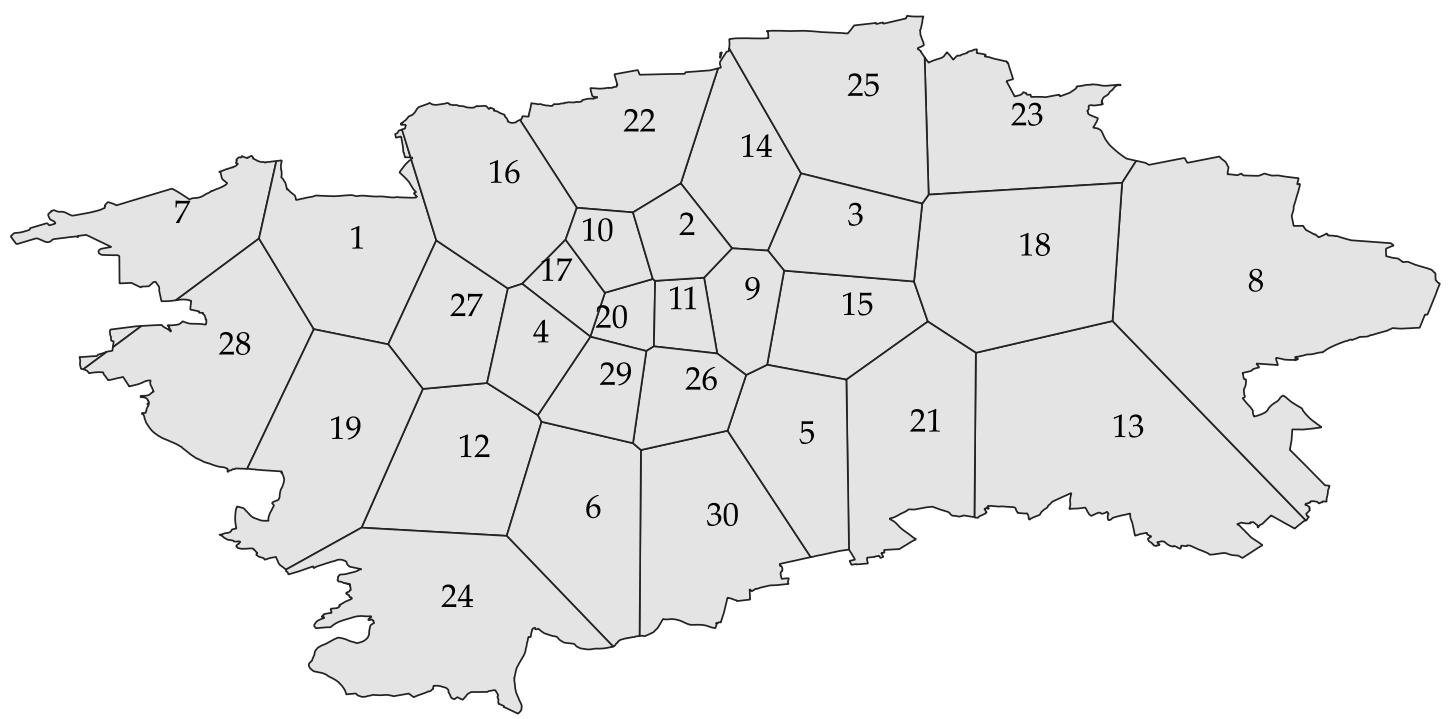

Note: This figure maps the boundaries of the city of Prague with locations defined by a kmeans-clustering procedure on GPS-locations of trip origins and depicted as Voronoi cells that contain the clustered points. Displayed index values correspond to indices used in the paper.

Figure 19: Weekly Waiting Times and Number of Drivers Compared to Prices

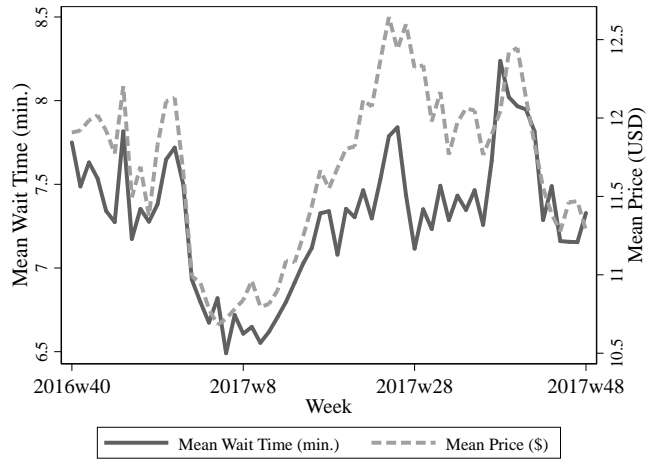

(a) Average Prices

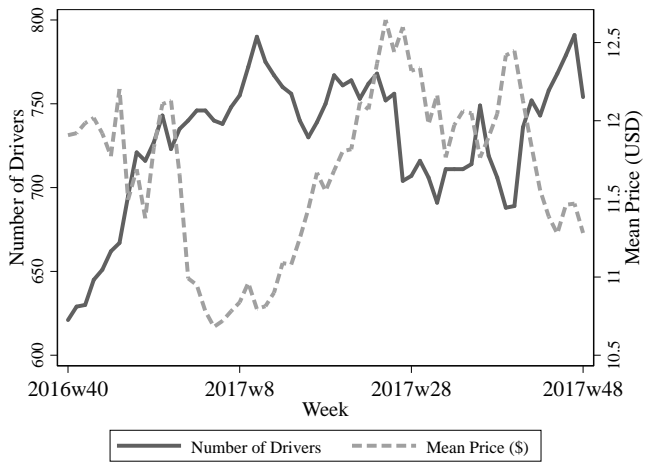

(b) Price and Driver Count 


\section{E Estimation Details}

\section{E.1 Recovering Latent Types: Conditional K-Means-Clustering Pro- cedure}

The clustering procedure allocates passengers to initial latent classes using a " $k$-means ++ " algorithm and then reallocates passenger types to best explain individual choices after controlling for observable features of the environment. This approach is based on Bonhomme et al. (2017) and uses Julia's Clustering package implementation of $k$-means ++ . Our discussion here is mainly based on the two.

\section{E.2 Location Parameter Estimates}

Here we offer additional details on location-specific parameter estimates. Figure 20 plots these estimates across origins and destinations.

Figure 20: Location-Specific Coefficient Estimates

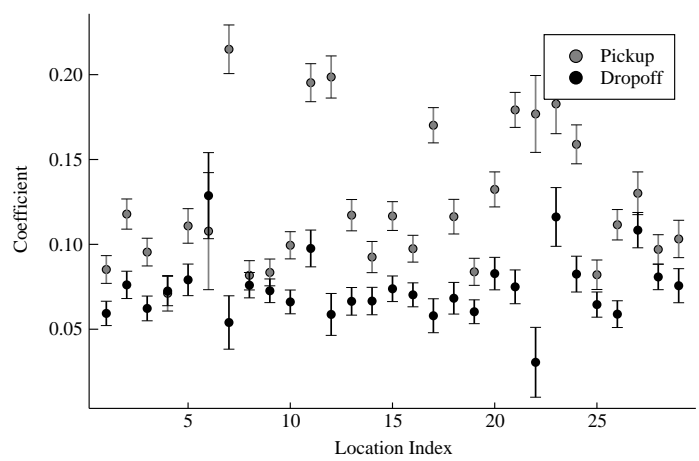

(a) Waiting Times $\times$ Locations

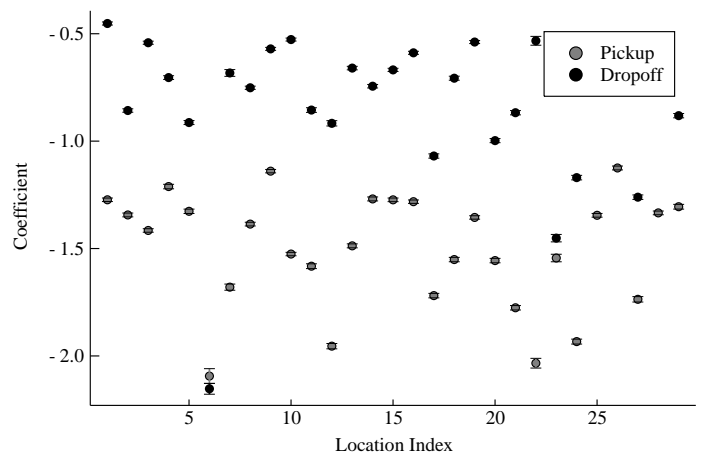

(b) Location Means

Note: This figure shows coefficient estimates for 120 location-specific parameters omitted from Table 2. Standard errors are depicted as bars around each point. Location indices may be cross-references with Figure 18. Panel (a) displays results for the coefficients on the interaction between waiting time and location, and Panel (b) displays results for the coefficients on location indicators, which can be interpreted as location-specific utility shifters relative to location index 1 .

\section{E.3 Model Fit}

Figure 21 illustrates the model's ability to fit the observed choices in the data. For each trip and corresponding driver bids, we use our estimates to predict whether each customer will pick the outside-option and plot the average prediction for each week.

Figure 22 Demonstrates the model's ability to correctly predict specific choices across auctions. We do this in two ways: first we use our estimates to predict which 
Figure 21: Model Fit On Outside Option Selection

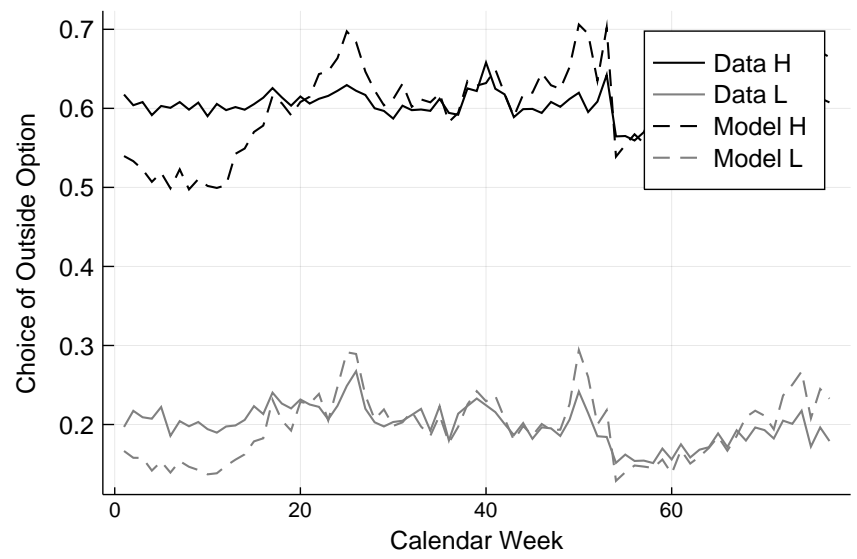

option each customer will pick inclusive of simulated draws of $\epsilon_{i, j, t}$. Because the $\epsilon_{i, j, t}$ represent unobservable attributes associated with each choice, this simulation will tend to add additional noise to our choice predictions. We also make the same predictions but set $\epsilon_{i, j, t}=0$ for all $i, j, t$, which improves our predictions by about $10 \%$.

Figure 22: Model Fit On Outside Option Selection

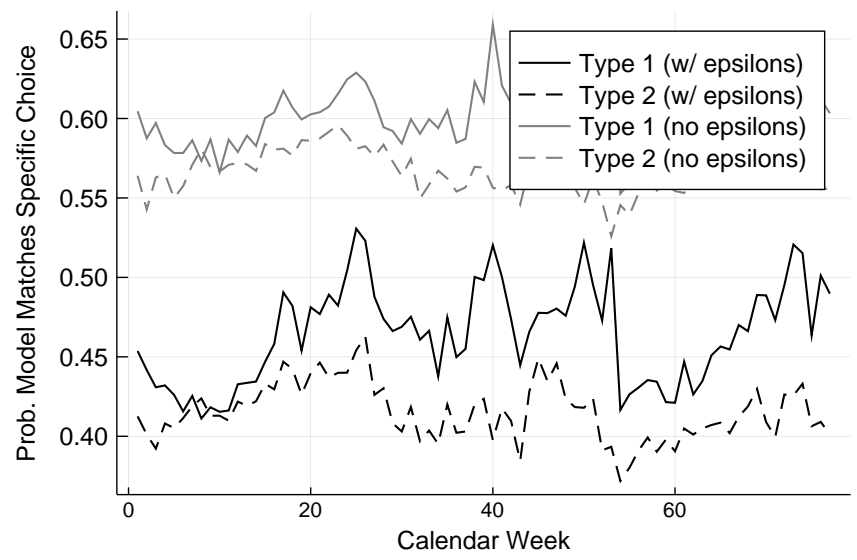

\section{E.4 Elasticities by Area}

See Table 9 and Table 10 below.

\section{E.5 Estimation Results Omitting Control Function}

Table 11 below presents the price and waiting time elasticity estimates in which the control function is omitted. 
Table 9: Bid Level Elasticities by Origin Area

\begin{tabular}{|c|c|c|c|c|}
\hline \multirow[b]{2}{*}{ ORIGIN AREA } & \multicolumn{2}{|c|}{ High VOT-Type Elasticities } & \multicolumn{2}{|c|}{ Low VOT-Type Elasticities } \\
\hline & PRICE & Waiting Time & Price & WAITING TIME \\
\hline 1 & -4.82 & -0.98 & -5.22 & -0.93 \\
\hline 2 & -2.71 & -0.37 & -3.66 & -0.3 \\
\hline 3 & -3.98 & -0.56 & -4.26 & -0.46 \\
\hline 4 & -2.95 & -0.38 & -3.79 & -0.29 \\
\hline 5 & -4.47 & -0.37 & -4.53 & -0.38 \\
\hline 6 & -5.05 & -0.59 & -5.37 & -0.57 \\
\hline 7 & -9.64 & -0.27 & -9.7 & -0.22 \\
\hline 8 & -10.0 & -0.55 & -9.75 & -0.48 \\
\hline 9 & -2.78 & -0.46 & -3.38 & -0.41 \\
\hline 10 & -2.62 & -0.33 & -3.7 & -0.25 \\
\hline 11 & -2.41 & -0.36 & -3.19 & -0.26 \\
\hline 12 & -5.26 & -0.47 & -5.55 & -0.43 \\
\hline 13 & -7.81 & -0.47 & -7.58 & -0.44 \\
\hline 14 & -3.95 & -0.51 & -4.34 & -0.44 \\
\hline 15 & -3.45 & -0.49 & -3.69 & -0.44 \\
\hline 16 & -3.69 & -0.38 & -4.54 & -0.31 \\
\hline 17 & -2.79 & -0.34 & -3.89 & -0.24 \\
\hline 18 & -6.75 & -0.78 & -6.45 & -0.7 \\
\hline 19 & -5.63 & -0.56 & -5.93 & -0.53 \\
\hline 20 & -2.52 & -0.31 & -3.43 & -0.22 \\
\hline 21 & -5.48 & -0.52 & -5.41 & -0.47 \\
\hline 22 & -5.06 & -0.7 & -5.45 & -0.6 \\
\hline 23 & -6.9 & -0.64 & -7.09 & -0.6 \\
\hline 24 & -10.31 & -0.44 & -9.6 & -0.38 \\
\hline 25 & -5.82 & -0.44 & -6.17 & -0.41 \\
\hline 26 & -2.98 & -0.39 & -3.59 & -0.34 \\
\hline 27 & -3.89 & -0.56 & -4.33 & -0.5 \\
\hline 28 & -6.66 & -0.43 & -6.56 & -0.39 \\
\hline 29 & -2.84 & -0.36 & -3.64 & -0.29 \\
\hline 30 & -4.98 & -0.57 & -5.2 & -0.51 \\
\hline
\end{tabular}

Note: This table shows price and waiting time elasticities across the thirty different origin places separated by high and low VOT types. Figure 18 shows location indices on a map of Prague. 
Table 10: Bid Level Elasticities by Destination Area

\begin{tabular}{|c|c|c|c|c|}
\hline \multirow[b]{2}{*}{ Destination AREa } & \multicolumn{2}{|c|}{ High VOT-Type Elasticities } & \multicolumn{2}{|c|}{ Low VOT-Type Elasticities } \\
\hline & PRICE & Waiting Time & PRICE & Waiting Time \\
\hline 1 & -4.3 & -0.47 & -4.44 & -0.42 \\
\hline 2 & -2.45 & -0.47 & -3.09 & -0.45 \\
\hline 3 & -3.6 & -0.32 & -3.88 & -0.26 \\
\hline 4 & -2.67 & -0.45 & -3.31 & -0.47 \\
\hline 5 & -4.16 & -0.43 & -4.39 & -0.32 \\
\hline 6 & -4.75 & -0.28 & -4.96 & -0.21 \\
\hline 7 & -9.35 & -0.38 & -8.33 & -0.39 \\
\hline 8 & -8.75 & -0.32 & -8.48 & -0.28 \\
\hline 9 & -2.64 & -0.37 & -3.16 & -0.36 \\
\hline 10 & -2.52 & -0.44 & -3.29 & -0.45 \\
\hline 11 & -2.32 & -0.35 & -2.87 & -0.35 \\
\hline 12 & -4.81 & -0.34 & -4.69 & -0.24 \\
\hline 13 & -7.02 & -0.26 & -7.06 & -0.21 \\
\hline 14 & -3.55 & -0.32 & -3.93 & -0.27 \\
\hline 15 & -3.18 & -0.32 & -3.51 & -0.3 \\
\hline 16 & -3.18 & -0.41 & -3.75 & -0.37 \\
\hline 17 & -2.57 & -0.45 & -3.41 & -0.46 \\
\hline 18 & -5.86 & -0.3 & -5.98 & -0.24 \\
\hline 19 & -5.24 & -0.33 & -5.13 & -0.26 \\
\hline 20 & -2.42 & -0.48 & -3.26 & -0.5 \\
\hline 21 & -5.04 & -0.33 & -5.3 & -0.28 \\
\hline 22 & -4.27 & -0.31 & -4.37 & -0.23 \\
\hline 23 & -6.14 & -0.19 & -5.77 & -0.17 \\
\hline 24 & -8.21 & -0.38 & -7.69 & -0.31 \\
\hline 25 & -5.42 & -0.36 & -5.51 & -0.28 \\
\hline 26 & -2.84 & -0.41 & -3.24 & -0.37 \\
\hline 27 & -3.35 & -0.35 & -3.46 & -0.29 \\
\hline 28 & -6.0 & -0.37 & -5.84 & -0.27 \\
\hline 29 & -2.72 & -0.36 & -3.19 & -0.34 \\
\hline 30 & -4.58 & -0.33 & -4.67 & -0.26 \\
\hline
\end{tabular}

Note: This table shows price and waiting time elasticities across the thirty different destination places separated by high and low VOT types. Figure 18 shows location indices on a map of Prague. 
Table 11: Estimated Elasticities

\begin{tabular}{|c|c|c|c|c|c|}
\hline \multirow{2}{*}{ Time of Day } & \multirow{2}{*}{ Individual Type } & \multicolumn{2}{|c|}{ Bid Level Elasticities } & \multicolumn{2}{|c|}{ Order Level Elasticities } \\
\hline & & PRICE & Waiting Time & PRICE & WAiting Time \\
\hline \multirow{5}{*}{$\begin{array}{l}\text { Daytime } \\
\text { 6am-6pm }\end{array}$} & Overall & -1.83 & -0.63 & -1.74 & -0.64 \\
\hline & H Price, H Wait Sensitivity & -4.08 & -1.18 & -3.83 & -1.09 \\
\hline & H Price, L Wait Sensitivity & -0.95 & -0.51 & -1.04 & -0.53 \\
\hline & L Price, H Wait Sensitivity & -2.15 & -0.6 & -2.05 & -0.68 \\
\hline & L Price, L Wait Sensitivity & -0.65 & -0.3 & -0.75 & -0.38 \\
\hline \multirow{5}{*}{$\begin{array}{l}\text { Evening } \\
\text { 6pm-6am }\end{array}$} & Overall & -2.58 & -0.25 & -2.4 & -0.28 \\
\hline & H Price, H Wait Sensitivity & -4.32 & -0.39 & -4.12 & -0.4 \\
\hline & H Price, L Wait Sensitivity & -1.25 & -0.2 & -1.33 & -0.22 \\
\hline & L Price, H Wait Sensitivity & -3.09 & -0.26 & -2.87 & -0.31 \\
\hline & L Price, L Wait Sensitivity & -1.04 & -0.15 & -1.12 & -0.18 \\
\hline
\end{tabular}

NoTE: This table shows the demand elasticity of price and waiting time across daytime and evening hours and individual type groupings. This table replicates Table 3 except that the model is estimated with no control function. We distinguish as high $(H)$ price sensitivity individuals who have below median values for $\beta_{i}^{p}$ and low $(L)$ price sensitivity individuals as those with above median values for $\beta_{i}^{p}$, and similarly for waiting time sensitivity. The first two columns show these elasticities among competing bids, reflecting the change in demand due to a $1 \%$ change in price or waiting time on a single bid. The second two columns show them with respect to choosing the outside option, reflecting a change in demand due to a $1 \%$ change in price or waiting time on all bids.

\section{E.6 Trip-Specific Heterogeneity Results}

The baseline results in Table 4 show the average nvoT across different hours of the day. Within each time-of-day and within each individual's type, however, there is additional heterogeneity due to the fact that some trips are more or less time-sensitive. For example, a given person may express higher NVOT if she is late for an appointment compared.

To analyze this type of heterogeneity, we define time-sensitive trips as the subset of trips in which a rider, having requested a ride, is faced with a set of bids in which (1) the arrival time falls around a rounded hour increment such as 9:00am or 2:00pm, (2) only bid would provide a trip that arrives before the hour and all others would provide a trip which arrive after the hour, and (3) a trip occurs on the platform. Because the NVOT estimated on trips generated on this subset is inherently selected due to point (3), we compare this against a similar subset of trips around a placebo clock-time. We thus define placebo trips by selecting orders where the arrival time falls around a clock time ending in :23 or :53, such as $8: 23 \mathrm{am}$ or $2: 53 \mathrm{pm}$. We then apply criteria (2) and (3) to these trips. The difference NVOT between time-sensitive and placebo trips reveals the relevant heterogeneity in time-sensitivity.

To augment our baseline results, Table 12 reports results for time sensitive trips and shows that the NVOT for this subset is about $58 \%$ greater than a comparable measure in the placebo group. The first column reports NvoT for all trips, as in Table 4, as a 
Table 12: Trip-Specific Heterogeneity in NVOT

\begin{tabular}{lccc}
\hline & \multirow{2}{*}{ All Trips } & \multicolumn{2}{c}{ Time-Selected Trips } \\
& & Placebo & Time-Sensitive \\
\hline NVOT (\$/hour) & $\$ 13.47$ & $\$ 17.92$ & $\$ 28.34$ \\
$N$ Trips & $1,021,007$ & 86,786 & 62,229 \\
$N$ Individuals & 80,161 & 32,438 & 21,651
\end{tabular}

Note: This table shows baseline mean NVOT estimates in the first column. The second column reports mean NVOT estimates for trips in which exactly one bid occurs before the 23rd and 53rd minute of each hour and additional bids fall beyond this time. The third column similarly reports NVOT estimates for trips in which exactly one bid occurs before the first minute of each hour. All estimates are presented in US dollars.

comparison. Our baseline NVOT results, therefore, can be interpreted as averages across this type of trip-specific heterogeneity. 